\title{
Engineering a de novo transport tunnel
}

Jan Brezovsky ${ }^{\dagger, \|}$, Petra Babkova ${ }^{\dagger, \|}$, Oksana Degtjarik $^{\ddagger}$, Andrea Fortova ${ }^{\dagger}$, Artur Gora $^{\dagger, \S}$, Iuliia Iermak ${ }^{\ddagger}$, Pavlina Rezacova ${ }^{\rfloor}$, Pavel Dvorak ${ }^{\dagger}$, Ivana Kuta Smatanova ${ }^{\ddagger}$, Zbynek Prokop ${ }^{\dagger}$, Radka Chaloupkova ${ }^{\dagger}$ and Jiri Damborsky ${ }^{\dagger, *}$

${ }^{\dagger}$ Loschmidt Laboratories, Department of Experimental Biology and Research Centre for Toxic Compounds in the Environment RECETOX, Faculty of Science, Masaryk University, Kamenice 5/A13, 625 oo Brno, Czech Republic

${ }^{\ddagger}$ Faculty of Science, University of South Bohemia in Ceske Budejovice, Branisovska 1760, 37005 Ceske Budejovice and Center for Nanobiology and Structural Biology ASCR, Zamek 136, 37333 Nove Hrady, Czech Republic

${ }^{\rfloor}$Institute of Organic Chemistry and Biochemistry ASCR, v.v.i. Flemingovo nam. 2, 166 1o Prague 6 and Institute of Molecular Genetics of the ASCR, v.v.i. Videnska 1083, 14220 Prague 4, Czech Republic

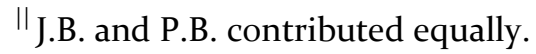

${ }^{\S}$ Present address: Biotechnology Centre, Silesian University of Technology, ul. Krzywoustego 8, 44-10o Gliwice, Poland

*jiri@chemi.muni.cz 
a

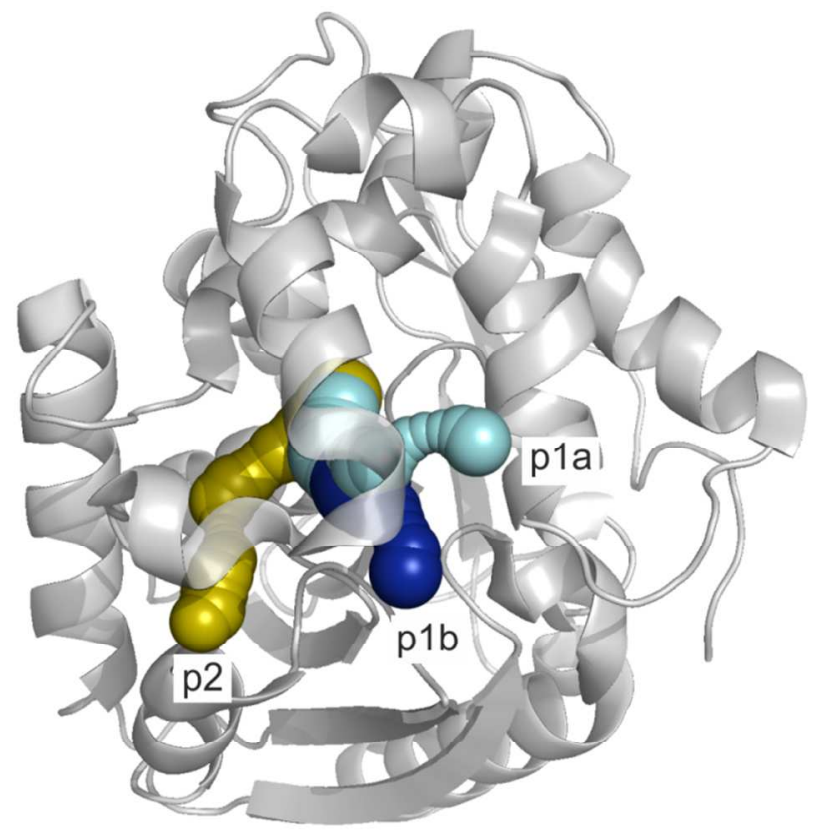

b

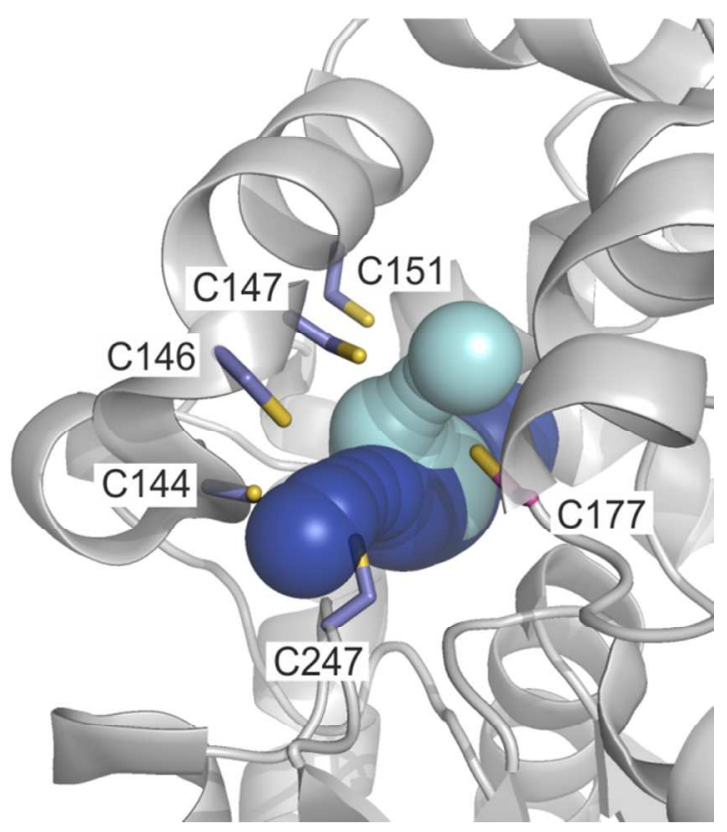

Figure S1. Transport tunnels of LinB-Wt and residues considered for closing of the main p1 tunnel. a) Transport tunnels identified in 12 available crystal structures of LinB-Wt using probe of $1 \AA$ Aadius. Tunnels p1a (cyan), p1b (blue) and p2 (dark yellow) are shown in sphere representation. The structure of LinB-Wt with representative tunnels (PDB-ID 1K63, see table S1) is show as a gray cartoon. b) Positions of cysteines considered for mutagenesis to form the disulfide bridge closing the $\mathrm{p} 1$ tunnel. Side-chain of substituted C177 is shown as magenta sticks. Mutable side-chains of residues facing the C177 mutated to cysteines are shown as blue sticks. Tunnels p1a (cyan) and p1b (blue) calculated by CAVER 3.0.2 are shown in sphere representation. See Table S2 for analysis of individual positions. 
a

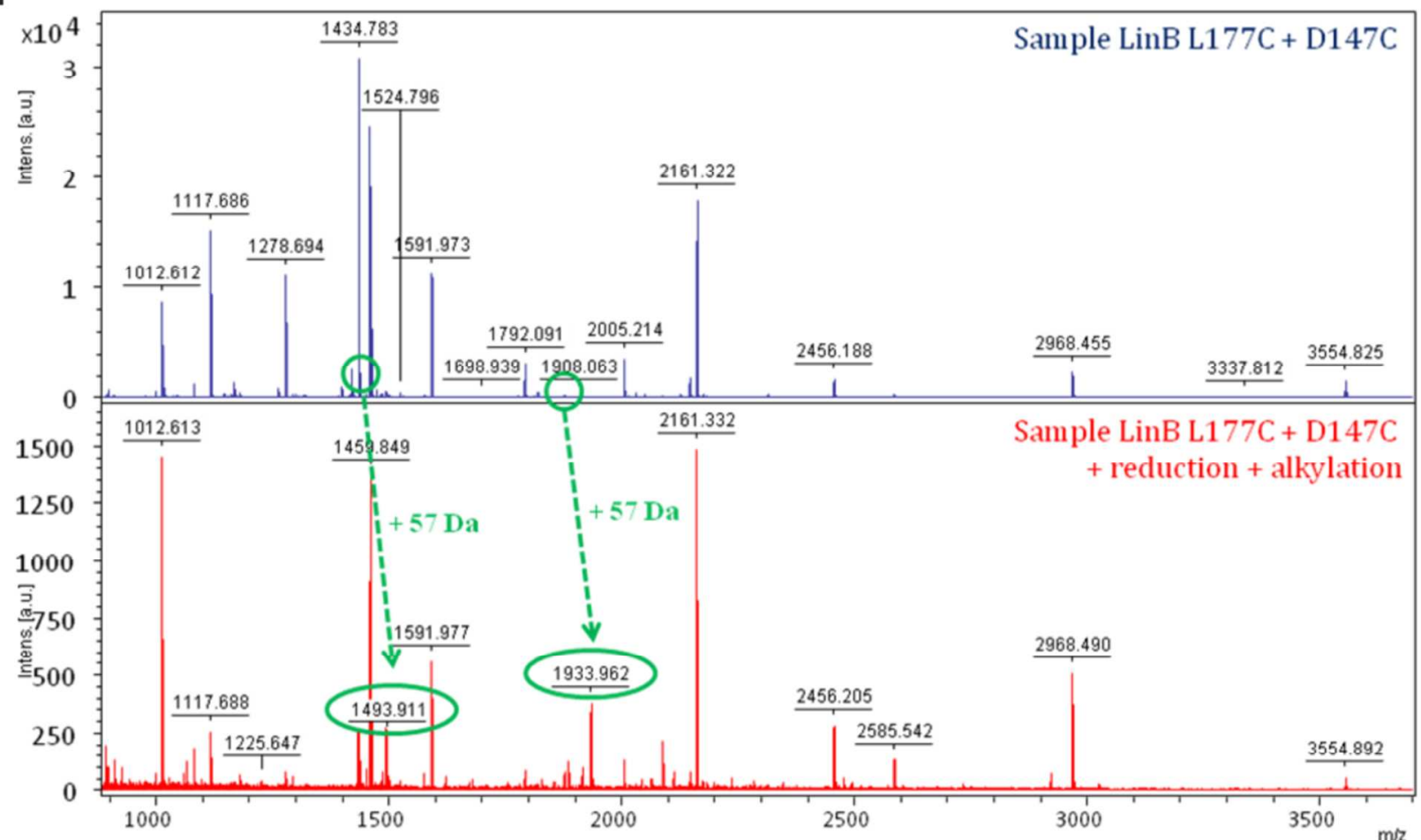

b

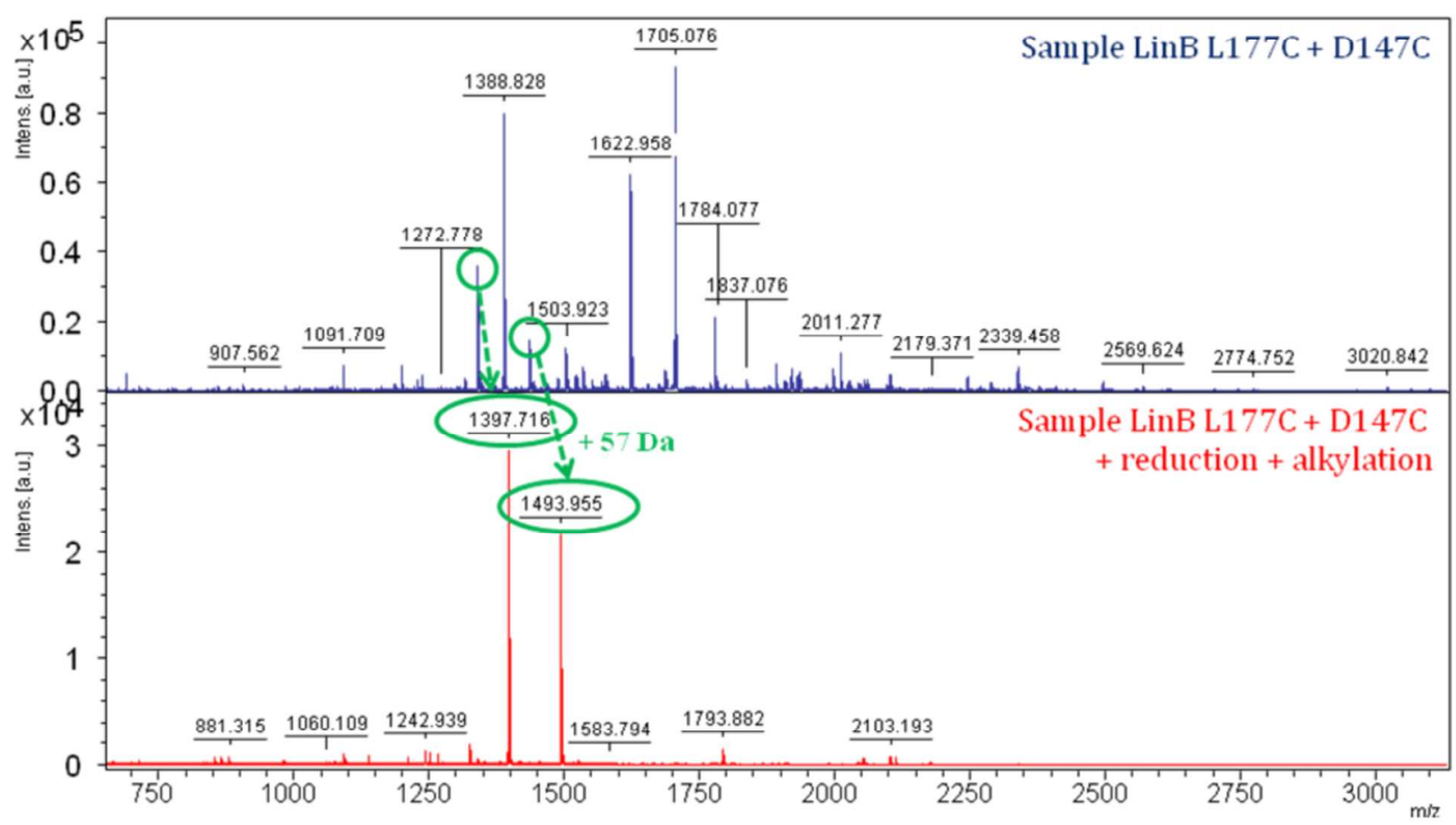

Figure S2. MALDI-MS/MS analysis of D147C+L177C mutant digested with trypsin and chymotrypsin. a) Spectra of trypsin digests before and after reduction and alkylation. b) Spectra of chymotrypsin digests before and after reduction and alkylation. 
a

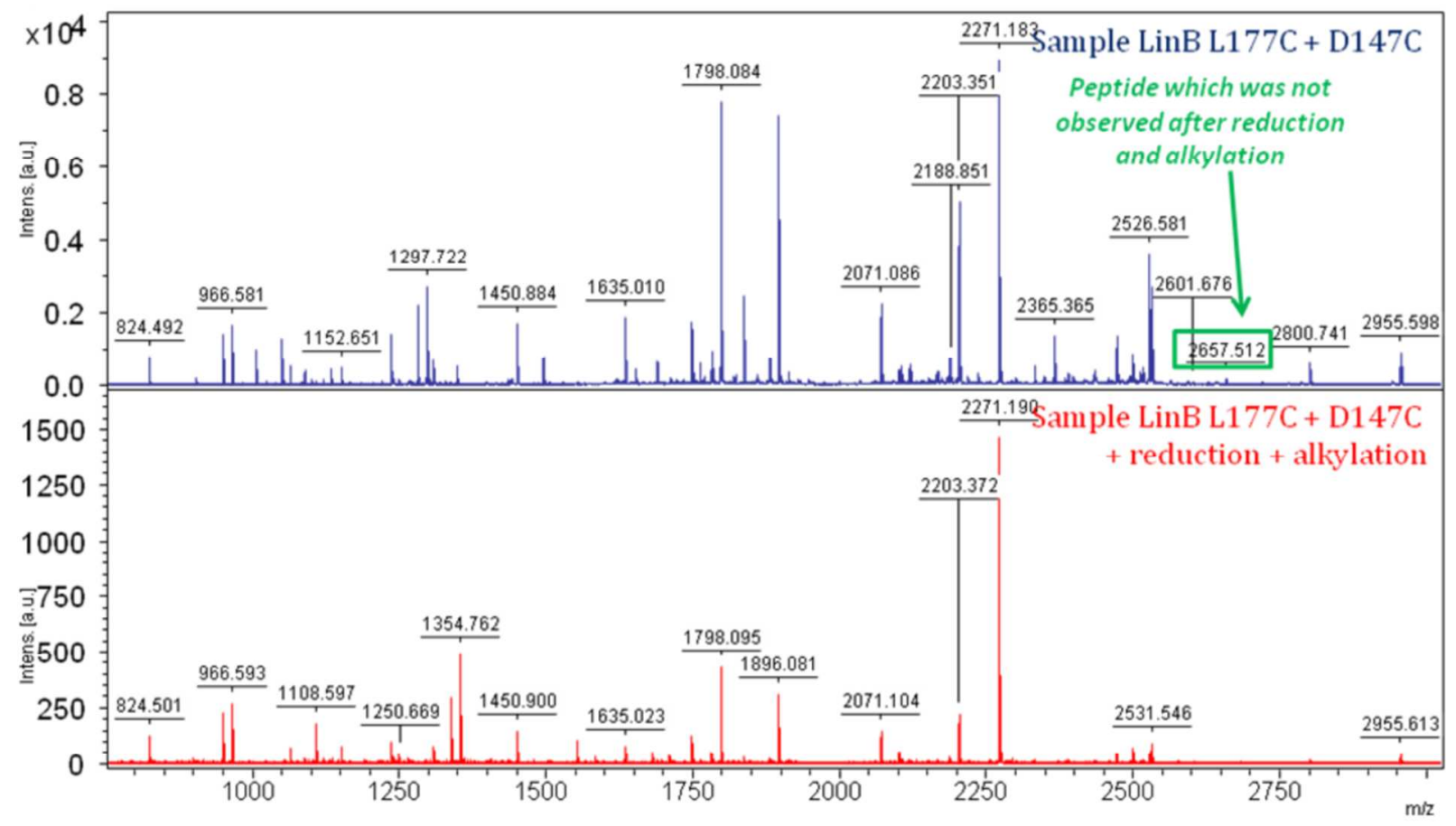

b

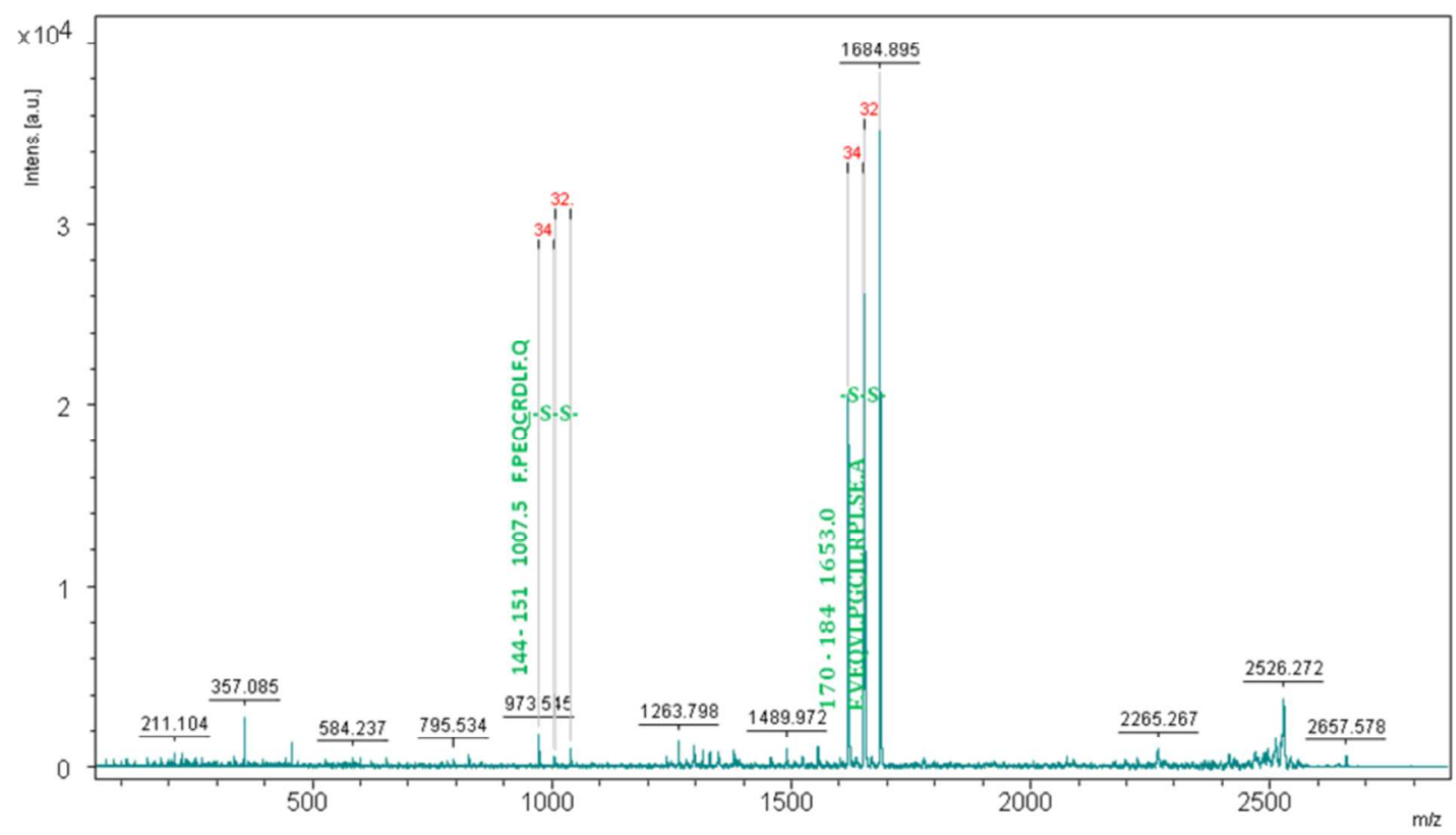

Figure S3. MALDI-MS/MS analysis of D147C+L177C mutant digested with pepsin. a) Spectra of trypsin digests before and after reduction and alkylation. b) MALDI-MS/MS analysis of peptide 2657.5 . 


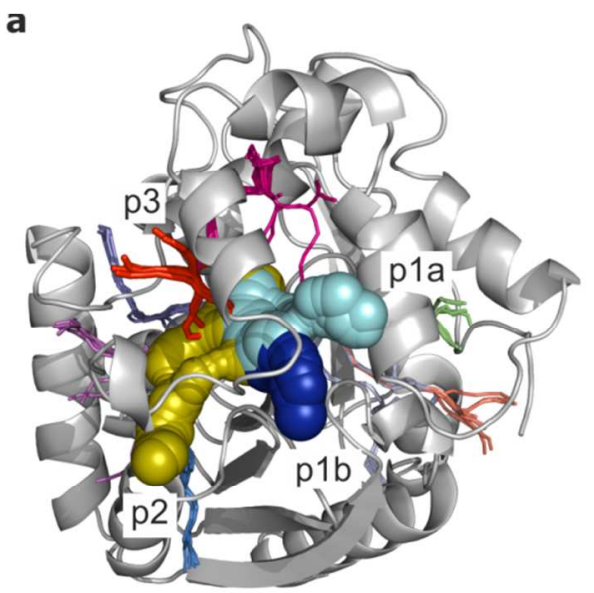

b

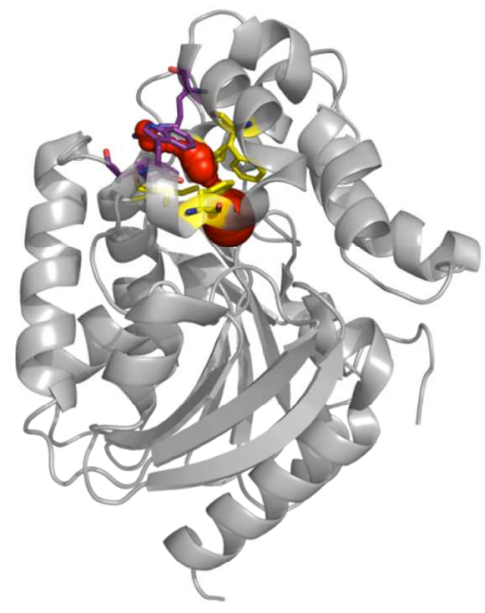

d

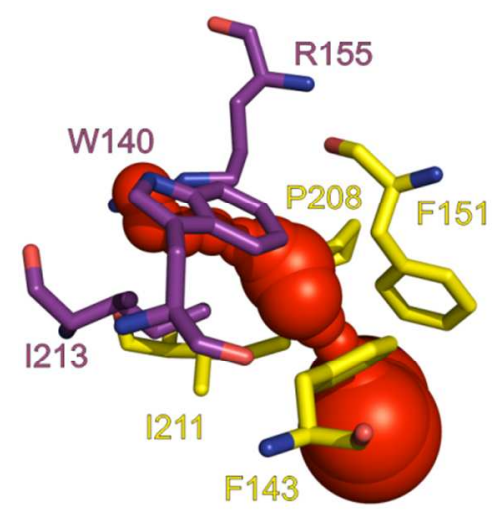

C

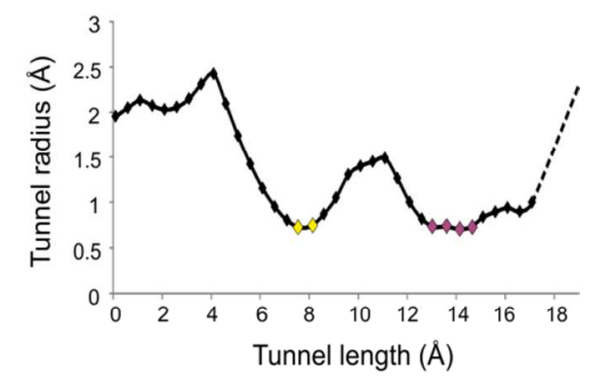

e

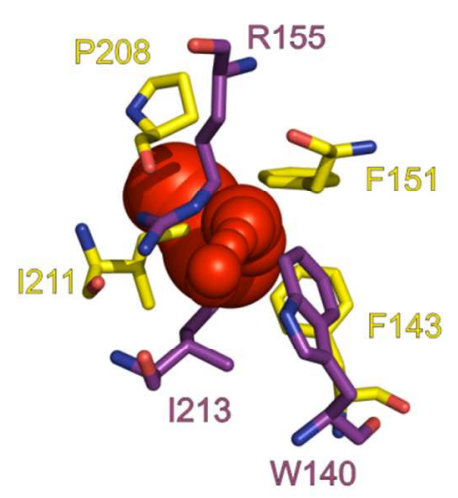

Figure S4. Location and characteristics of potential p3 pathway in LinB-Wt. a) Transport tunnels and potential pathways identified in 12 crystal structures of LinB-Wt using CAVER 3.0.2 with 0.6 ̊̊ radius probe. Tunnels p1a (cyan), p1b (blue) and p2 (dark yellow) are shown as spheres. The centerline of a pathway $\mathrm{p} 3$ selected for de novo introduction of a transport tunnel is indicated by red sticks. The remaining eight pathways are shown with their centerlines as lines. b) p3 tunnel (red spheres) in the structure of LinB-Wt (gray cartoon). The residues forming the bottlenecks 1 and 2 are shown as yellow and violet sticks, respectively. c) Profile of the $\mathrm{p} 3$ pathway. The positions of the bottlenecks 1 and 2 are represented by yellow and violet diamonds in the profile, respectively. The dashed line indicates further widening of the pathway at the protein surface. Detail of the p3 pathway and its bottleneck residues as a side-view (d) and oriented from the surface toward the active site (e). 


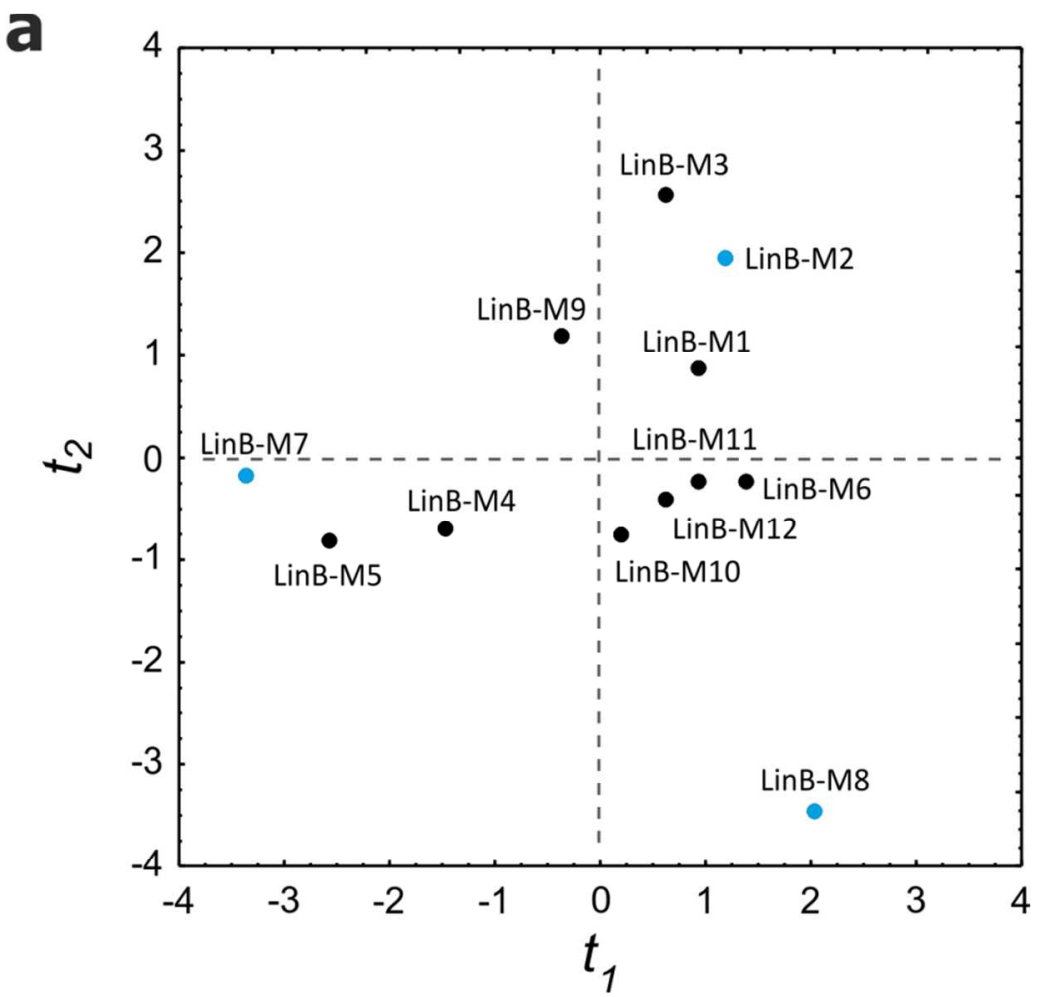

b

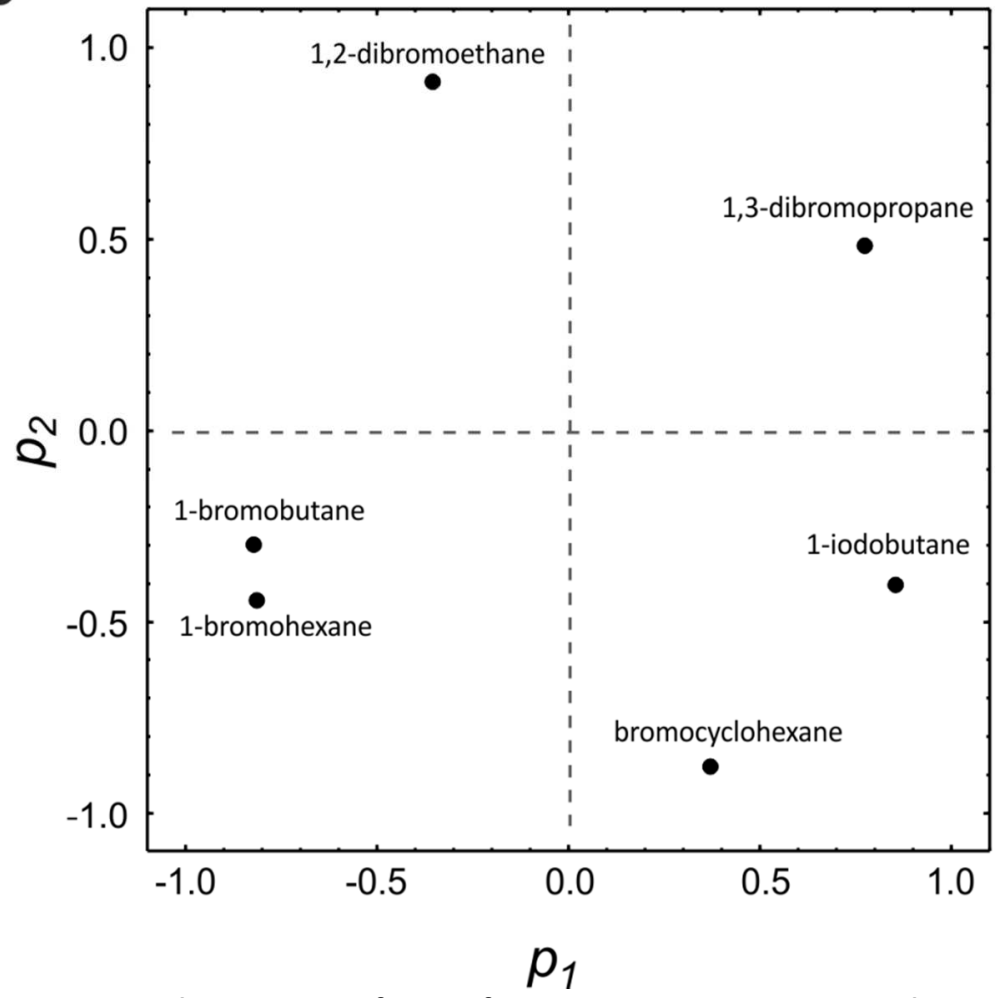

Figure S5. Substrate specificity of LinB variants M1-M12 with engineered p3 tunnel. a) Score plot $t_{1} / t_{2}$ from principal component analysis of substrate specificity data measured with six selected substrates. b) Corresponding loading plot $p_{1} / p_{2}$ from principal component analysis showing the substrates that govern the clustering of the enzymes in (a). The three mutants covering the whole substrate specificity space selected for further characterization are shown in blue. 

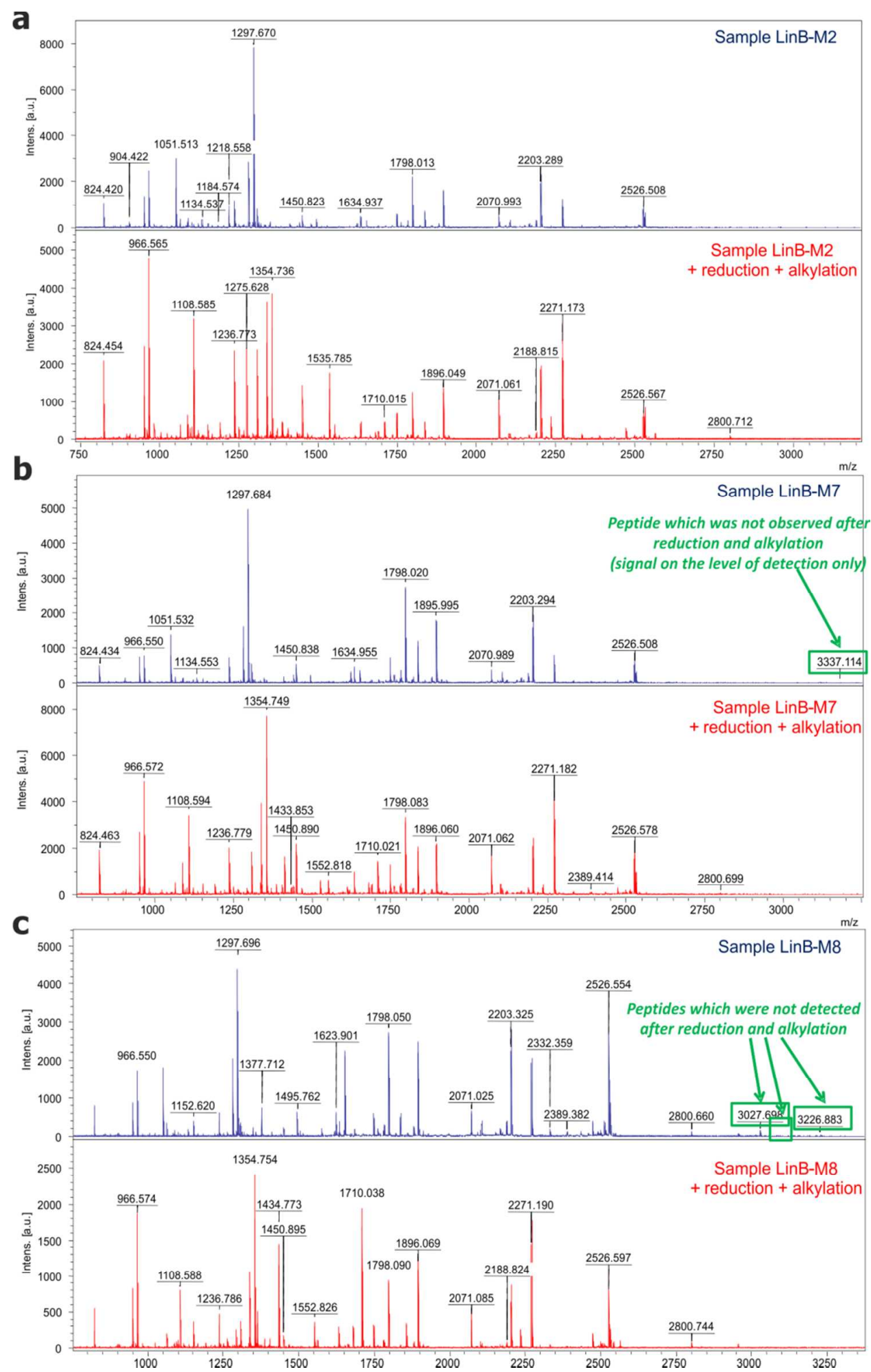

Figure S6. MALDI-MS/MS analysis of LinB variants $M 2, M 7$ and $M 8$ digested by pepsin before and after reduction and alkylation. a) Spectra of LinB-M2 mutant. b) Spectra of LinB-M7 mutant. c) Spectra of LinB-M8 mutant. 
a

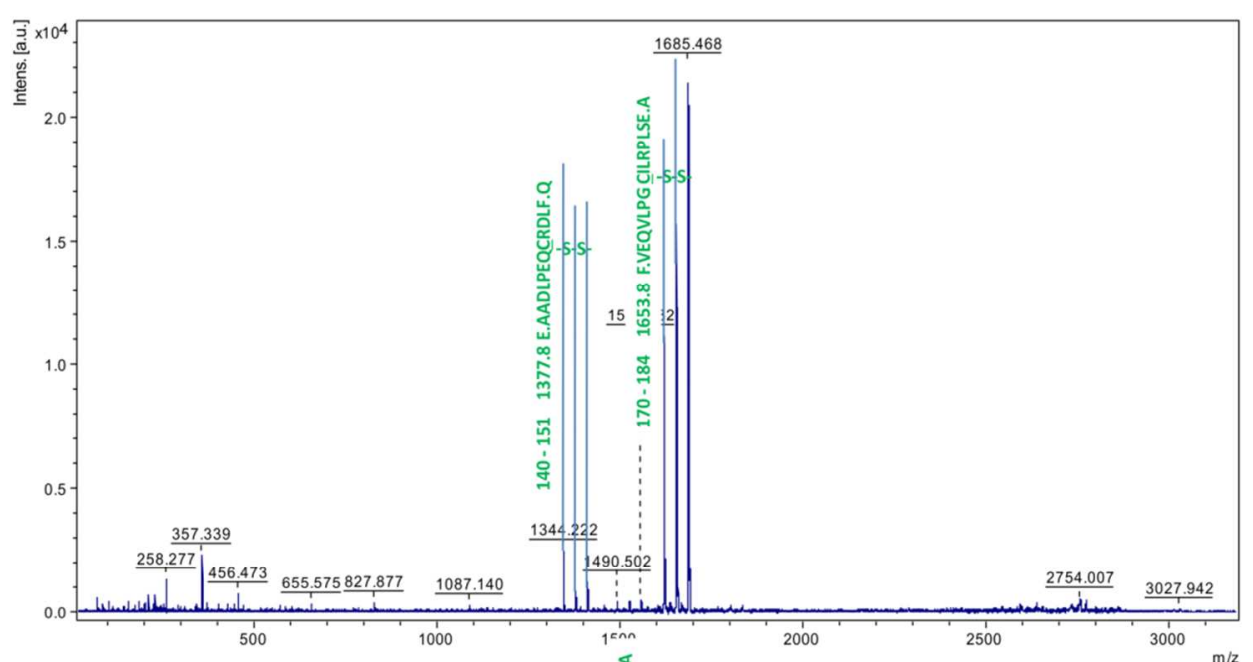

b

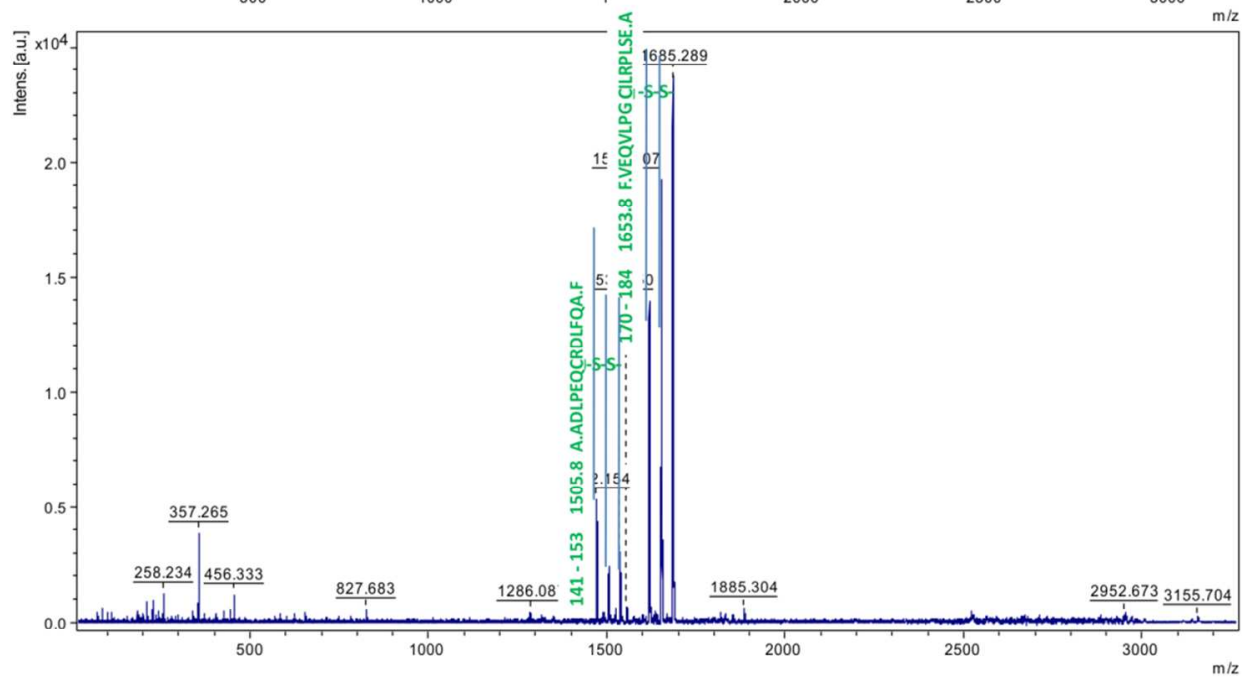

C

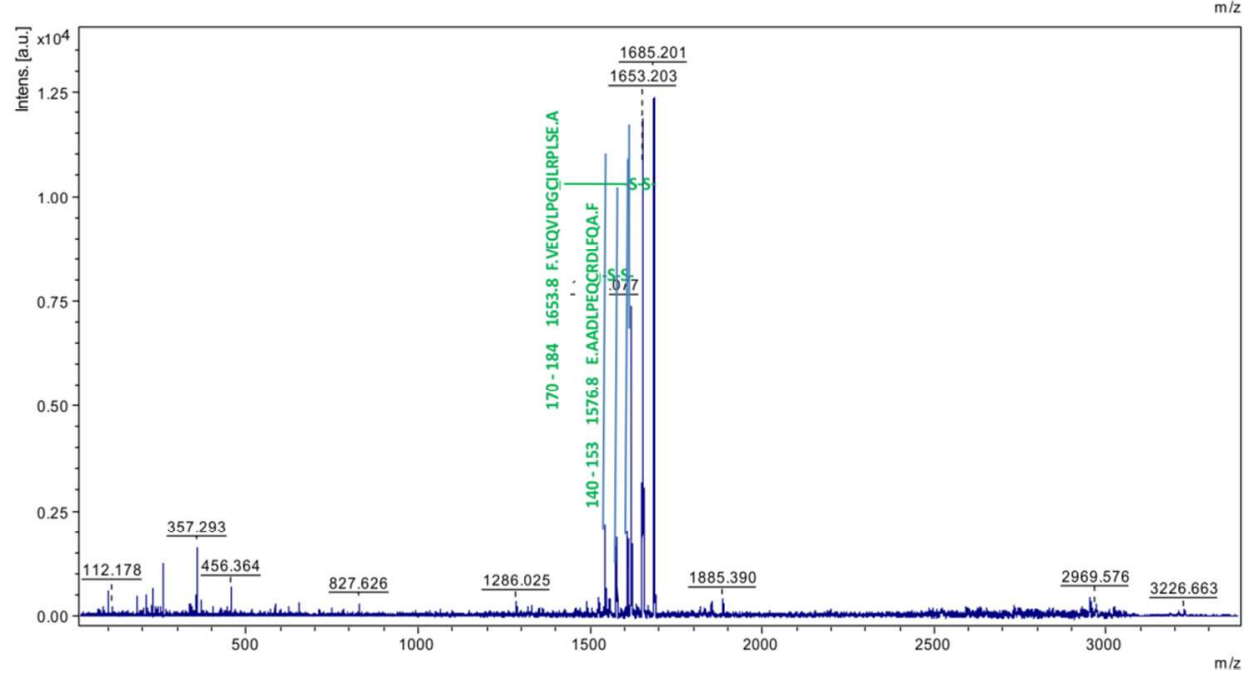

Figure S7. MALDI-MS/MS analysis of three identified peptides from LinB-M8 pepsin digest. a) Analysis of peptide 3027.7. b) Analysis of peptide 3155.7. c) Analysis of peptide 3226.7.

SB 


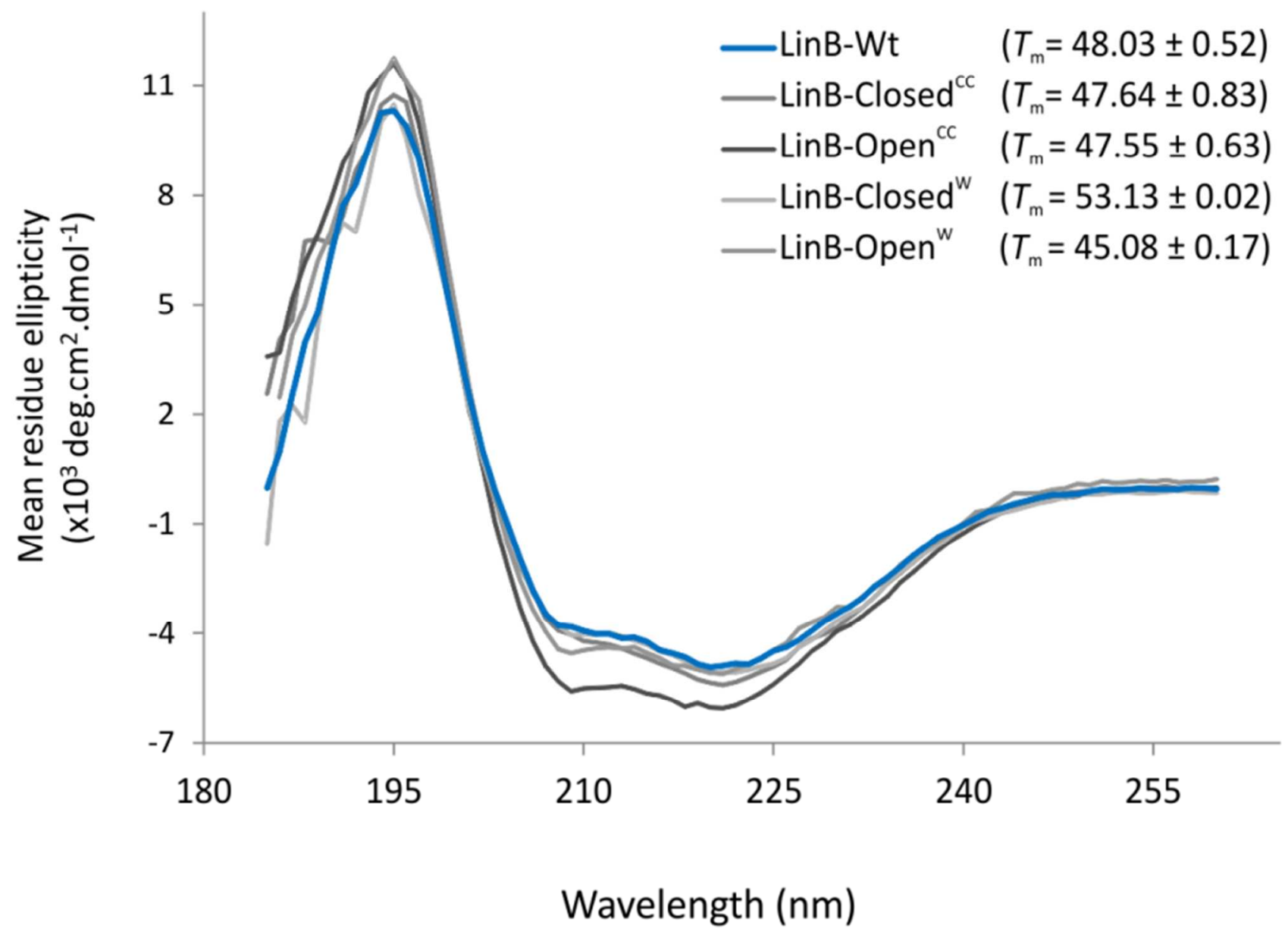

Figure S8. Far-UV circular dichroism spectra and melting temperatures $\left(T_{m}\right)$ of LinB variants. 
a
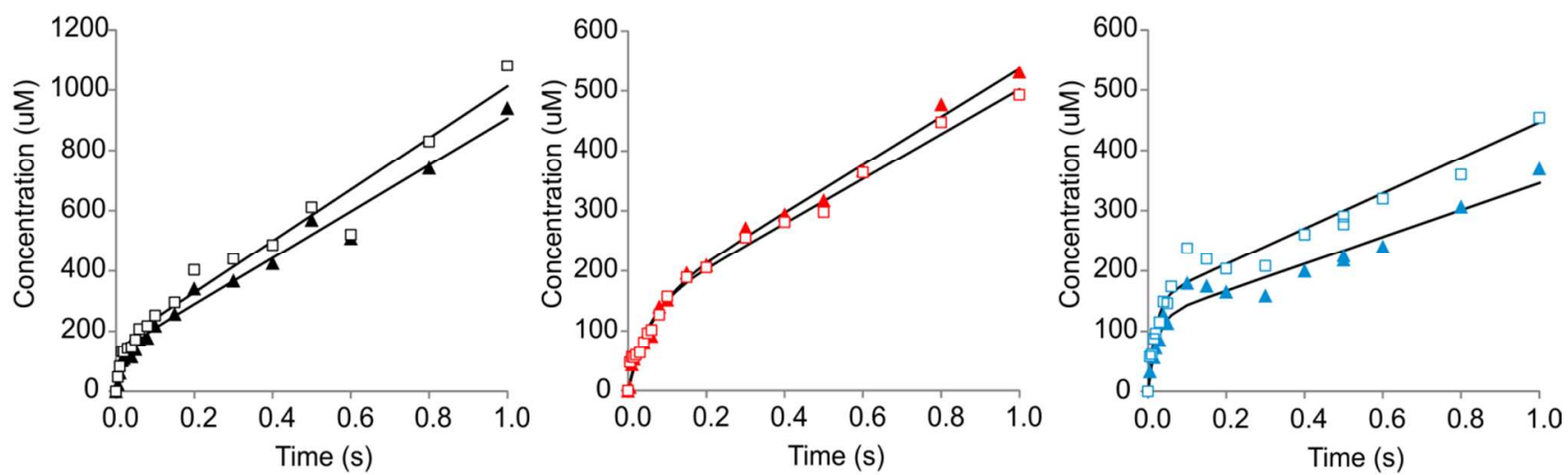

b
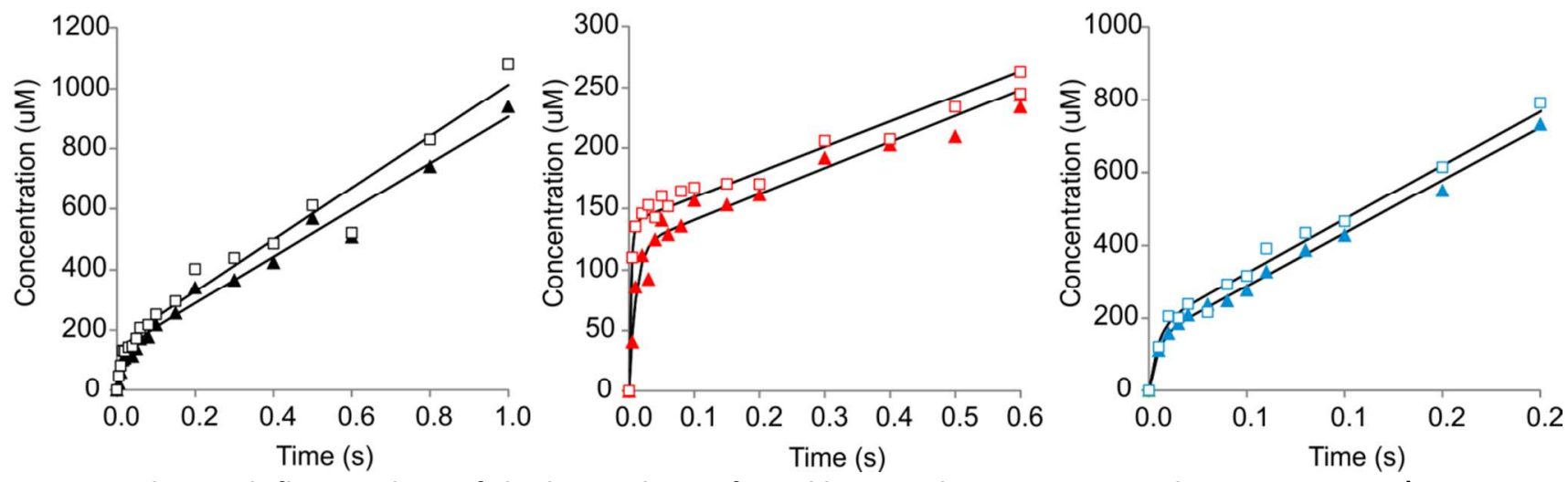

Figure S9. Rapid quench flow analysis of the burst phase of 1,2-dibromoethane reaction with LinB variants. a) Comparison of the reaction burst upon mixing of $8.00 \mathrm{mM}$ 1,2-dibromoethane with $160 \mu \mathrm{M}$ LinB-Wt (black), $9.67 \mathrm{mM}$ 1,2-dibromoethane with $140 \mu \mathrm{M}$ LinB-Closed ${ }^{C C}$ (red) and $7.77 \mathrm{mM}^{1,2-d i b r o m o e t h a n e ~ w i t h ~} 170 \mu \mathrm{M}$ LinB-Open ${ }^{\mathrm{CC}}$ (blue). b) Comparison of the reaction burst upon mixing of $8.00 \mathrm{mM}$ 1,2-dibromoethane with $160 \mu \mathrm{M}$ LinB-Wt (black), $6.19 \mathrm{mM}$ 1,2-dibromoethane with $164 \mu \mathrm{M}$ LinBClosed $^{\mathrm{W}}$ (red), and $6.71 \mathrm{mM}$ 1,2-dibromoethane with $143 \mu \mathrm{M}$ LinB-Open $^{\mathrm{W}}$ (blue). Halide ( $\square$ ) and alcohol ( $\mathbf{\Delta}$ ) product concentrations were analyzed by ion and gas chromatography, respectively. Solid lines represent the best fits to the kinetic data. Note different scales on both axes. 


\section{a}

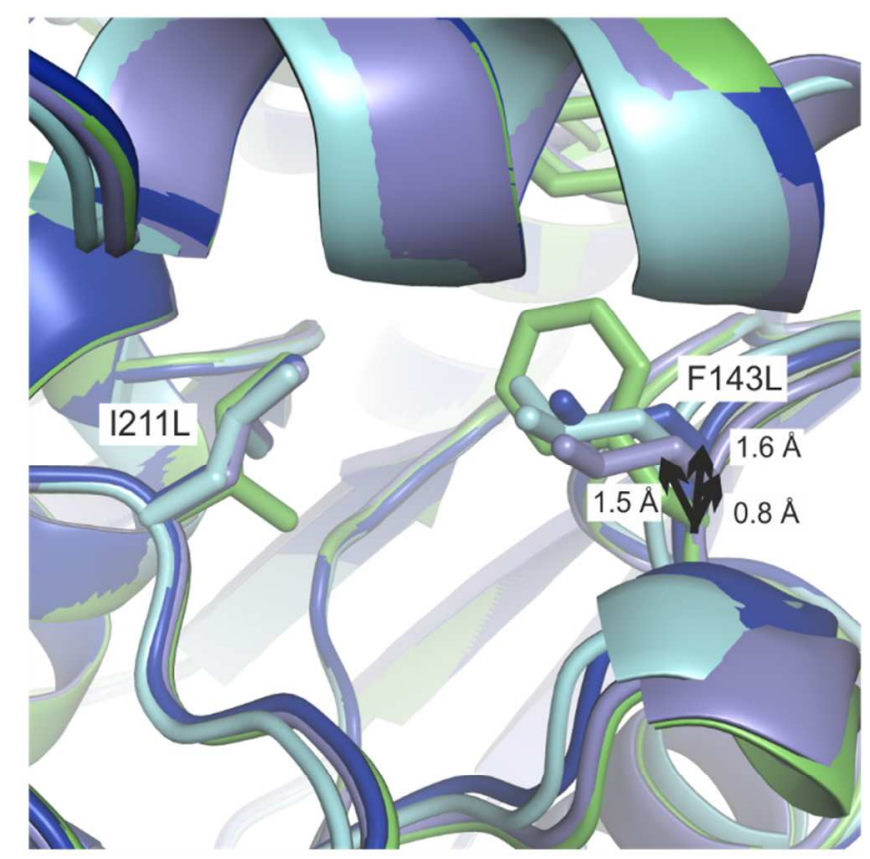

b
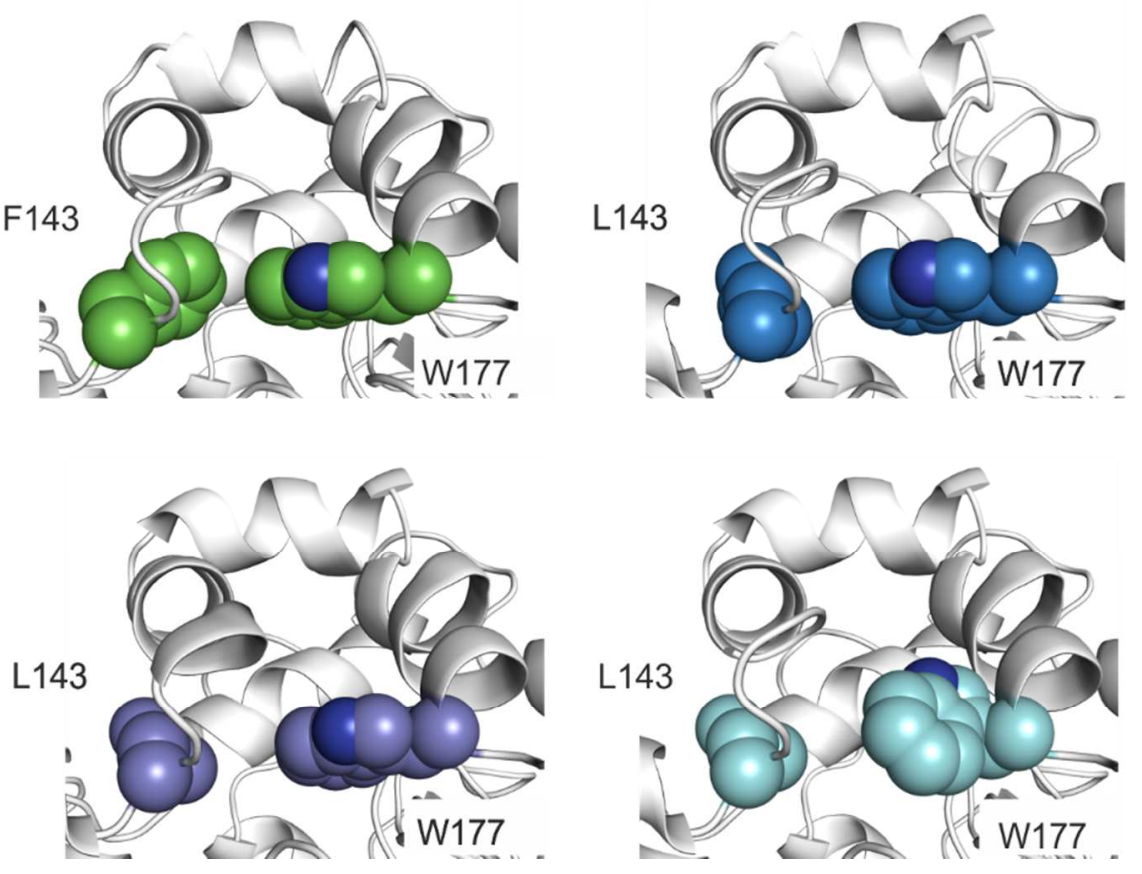

Figure S10. Structural consequences of $p 3$ tunnel opening in LinB-Open ${ }^{w}$. a) Backbone rearrangement in the region of the $p 3$ tunnel countering its opening in LinB-Open ${ }^{w}$. Template structure of LinB-Closed ${ }^{W}$ (PDB-ID 4WDQ) is shown in green, whereas structures of LinB-Open ${ }^{W}$ are shown in violet (PDB-ID 4WDR - chain A), cyan (PDB-ID 4WDR - chain B) and blue (PDB-ID 5LKA). The black arrows indicate the backbone movement in comparison to the arrangements in the template LinB-Closed ${ }^{\mathrm{W}}$. b) Conformations of $\mathrm{W} 177$ and $\mathrm{F}(\mathrm{L}) 143$ in LinB-Open ${ }^{\mathrm{W}}$ and LinB-Closed ${ }^{\mathrm{W}}$ variants. Proteins are shown as white cartoon; residues at the positions 177 and 143 are shown as green (LinB-Closed ${ }^{\text {w }}$; PDB-ID 4WDQ), violet (LinB-Open ${ }^{\text {; }}$; PDB-ID 4WDR - chain A), cyan (LinB-Open ${ }^{\text {}}$; PDB-ID 4WDR - chain B) and blue (LinB-Open ${ }^{\text {w }}$; PDB-ID 5LKA) spheres. 


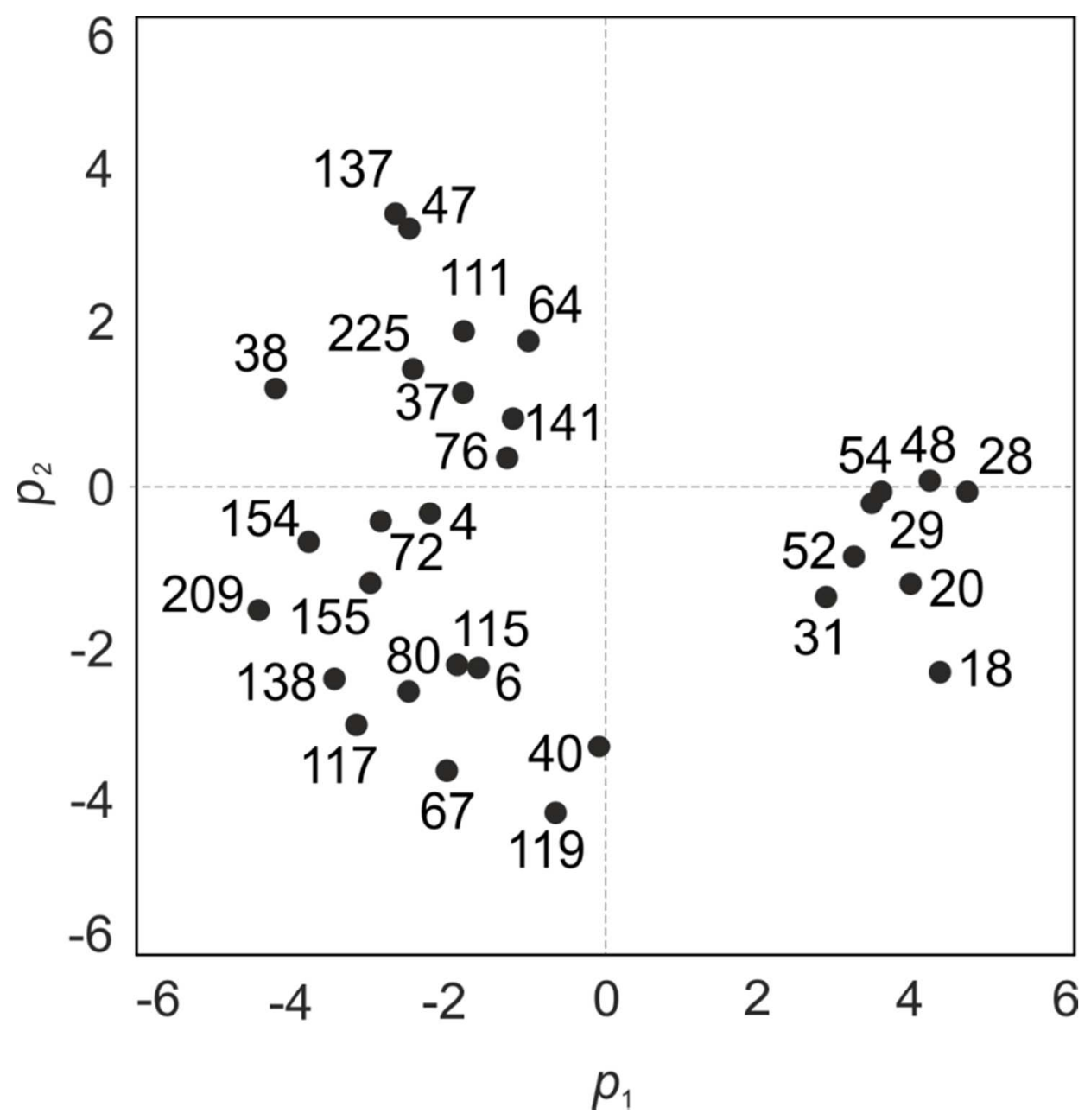

Figure S11. Loadings plot from Principal Component Analysis of substrate specificity data measured with the standard set of 30 halogenated substrates. The plot shows the substrates responsible for clustering of the enzymes in Figure $2 \mathrm{C}$ according to the contributions of the activities with individual substrates to principal component 1 and 2. The substrates are labelled as follows: 4: 1-chlorobutane; 6: 1-chlorohexane; 18: 1-bromobutane; 20: 1-bromohexane; 28: 1-iodopropane; 29: 1-iodobutane; 31: 1iodohexane; 37: 1,2-dichloroethane; 38: 1,3-dichloropropane; 40: 1,5-dichloro-pentane; 47: 1,2-di-bromoethane; 48: 1,3dibromopropane; 52: 1-bromo-3-chloropropane; 54: 1,3-diiodopropane; 64: 2-iodo-butane; 67: 1,2-dichloropropane; 72: 1,2dibromopropane; 76: 2-bromo-1-chloropropane; 80: 1,2,3-trichloropropane; 111: bis(2-chloro-ethyl)ether; 115: chlorocyclohexane; 117: bromocyclohexane; 119: (1-bromomethyl)cyclohexane; 137: 1-bromo-2-chloroethane; 138: chlorocyclopentane; 141: 4-bromobutyronitrile; 154: 1,2,3-tribromopropane; 155: 1,2-dibromo-3-chloropropane; 209: 3-chloro2-methylpropene; 225: 2,3-dichloro-propene. 


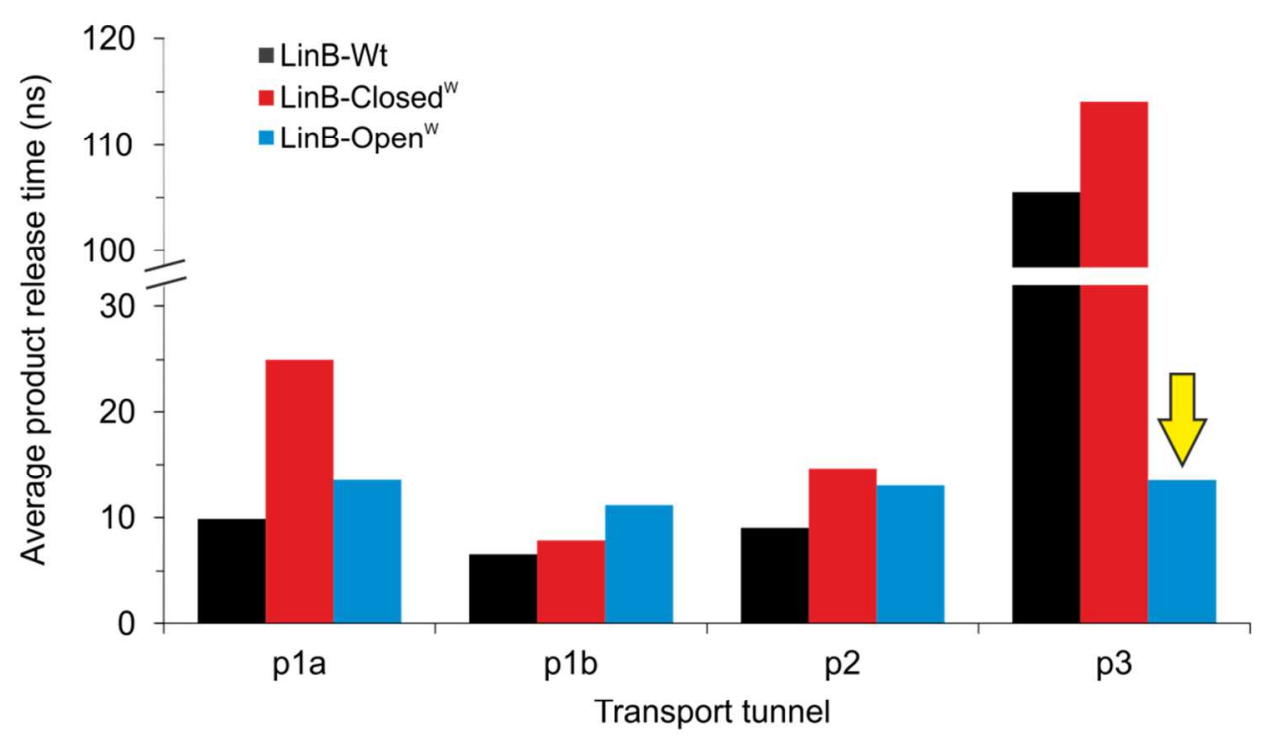

b

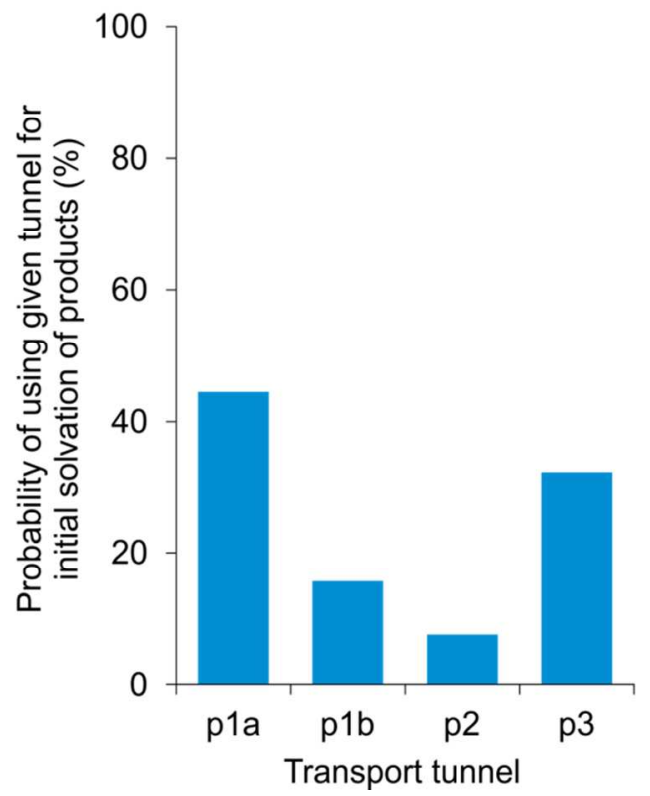

C

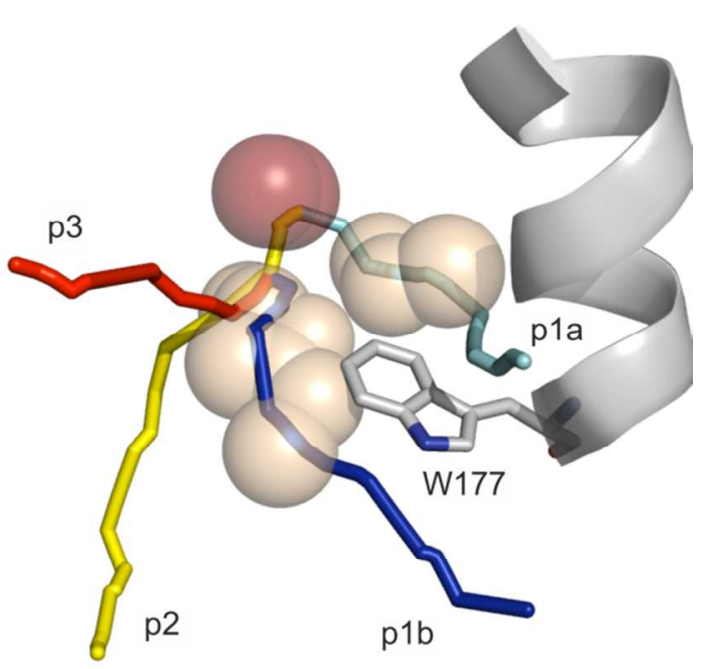

Figure S12. Effects of tunnel engineering on the products' release rates. a) Average times required for the release of both products from the active site of LinB variants via particular tunnel during accelerated molecular dynamics simulations. A change in the transport rates of products via p3 tunnel are highlighted by the yellow arrow. Note that due to the boosts applied during the simulations, the observed duration of the product release would generally correspond to three orders of magnitude longer periods. In the case of release via p3 tunnel in LinB-Wt and LinB-Closed ${ }^{\mathrm{W}}$, the release times represent lower bounds since a release of the bromide ion through this tunnel has never been observed within the simulation time of 200 ns and therefore the actual release times would likely be much larger. b) The probability of initial solvation of products via a particular tunnel in simulations of LinB-Open ${ }^{\mathrm{W}}$ leading to products leaving via pla tunnel. c) Most probable locations of bound products in tunnels of LinB-Open ${ }^{\mathrm{W}}$. Individual tunnels are shown as colored sticks, protein structure as gray cartoon, introduced W177 as gray sticks, bromide ions located between halide-stabilizing residues as red sphere and 2-bromoethanol as beige spheres. The most probable location of the alcohol products corresponds to $\mathrm{p} 1 \mathrm{a}$ and $\mathrm{p} 1 \mathrm{~b}$ tunnels, likely resulting in their partial blockage. 
a

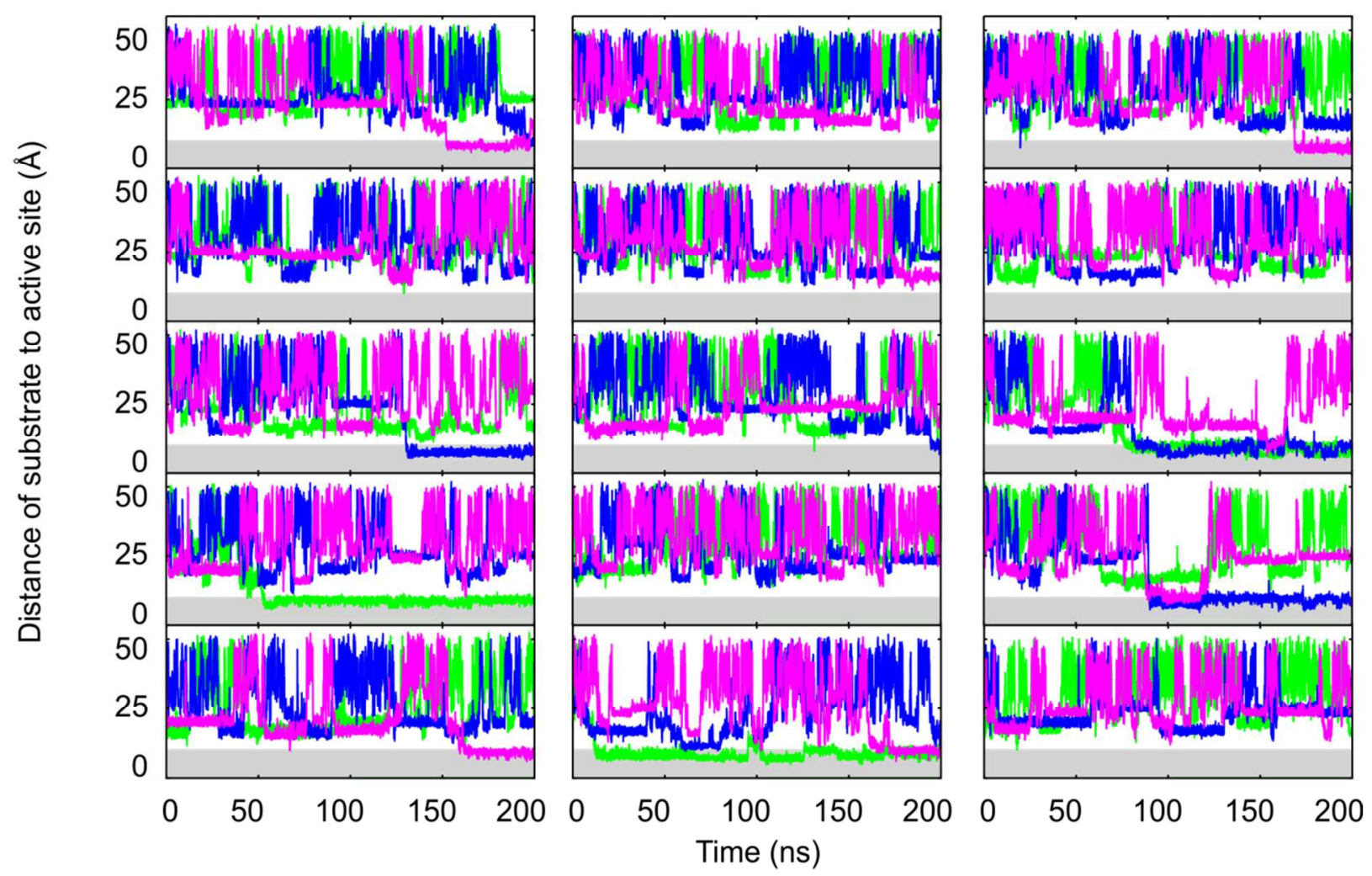

b

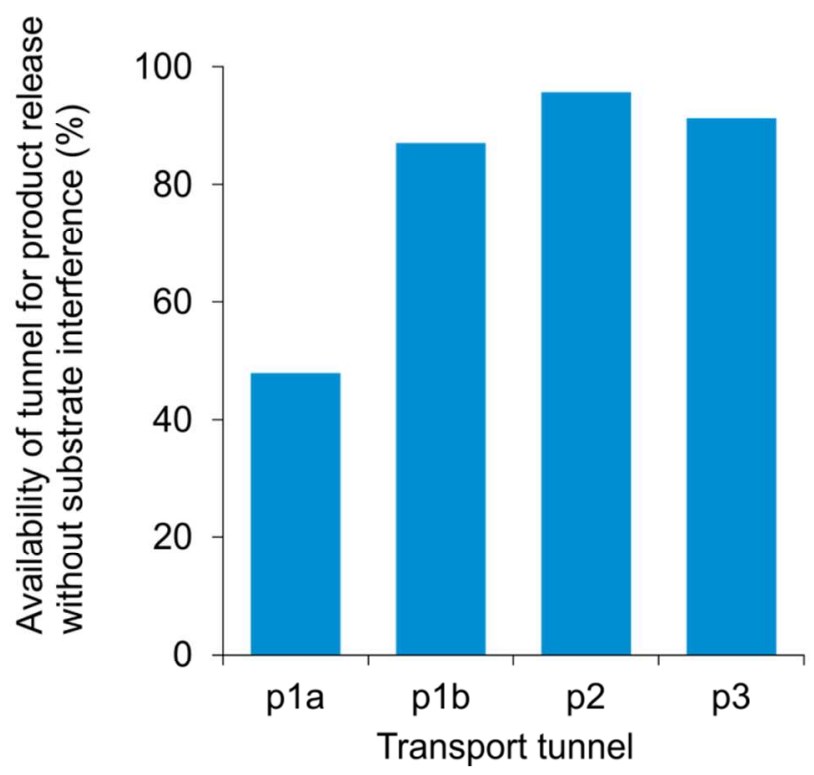

Figure S13. Potential interference of a substrate entry with the product release in LinB-Open ${ }^{\mathrm{w}}$. a) Evolution of distances of three substrate (1,2-dibromoethane) molecules from the active site of LinB-Open ${ }^{\text {w }}$, which corresponds to the gray zone in the graphs. Different colors represent individual substrate molecules. b) Likelihood of products (2bromoehanol and bromide ion) being released via a particular tunnel without interference from the substrate occuping this tunnel. 
Table S1. Geometry of the transport tunnels identified in 12 crystal structures of LinB-Wt available in the Protein Data Bank.

\begin{tabular}{|c|c|c|c|c|c|c|c|c|c|c|c|c|c|c|}
\hline PDB-ID & & 1CV2 & 1D07 & $1 \mathrm{G} 42$ & 1G4H & 1G5F & $1 / 27$ & $1 / Z 8$ & 1K5P & $1 \mathrm{~K} 63$ & 1K6E & 1MJ5 & 2BFN & Average \\
\hline \multirow{3}{*}{$\begin{array}{l}\text { Bottleneck } \\
\text { radius (Å) }\end{array}$} & p1b & 1.4 & 1.4 & 1.1 & 1.2 & 1.2 & 1.6 & 1.5 & 1.7 & 1.5 & 1.5 & 1.5 & 1.5 & $1.4 \pm 0.2$ \\
\hline & p1a & 1.0 & 1.2 & 1.3 & 1.3 & 1.3 & 1.0 & 1.2 & 1.2 & 1.1 & 1.1 & 0.8 & 0.9 & $1.1 \pm 0.2$ \\
\hline & p2 & 1.0 & 1.1 & 1.0 & 0.9 & 0.9 & 0.9 & 1.0 & 1.1 & 1.0 & 1.0 & 1.0 & 1.0 & $1.0 \pm 0.1$ \\
\hline \multirow{3}{*}{ Length ( $\AA$ ) } & p1b & 13 & 13 & 14 & 12 & 13 & 15 & 13 & 13 & 14 & 12 & 14 & 13 & $13 \pm 1$ \\
\hline & p1a & 16 & 14 & 16 & 15 & 15 & 16 & 16 & 17 & 16 & 15 & 19 & 17 & $16 \pm 1$ \\
\hline & p2 & 19 & 19 & 20 & 20 & 20 & 20 & 20 & 19 & 18 & 19 & 18 & 19 & $19 \pm 1$ \\
\hline
\end{tabular}


Table S2. Bottleneck residues of p1a and p1b tunnels in LinB-Wt.

\begin{tabular}{|c|c|c|c|c|}
\hline Residue & Location $^{a}$ & Side-chain orientation & Mutability score $^{\text {b }}$ & Distance SG-SG $(\AA ̊ \AA){ }^{c}$ \\
\hline F143 & \multirow{10}{*}{ across tunnel } & along tunnel & 8 & N.A. \\
\hline P144 & & toward tunnel & 5 & 7.0 \\
\hline E145 & & toward solvent & 9 & N.A. \\
\hline Q146 & & toward tunnel & 9 & 6.1 \\
\hline D147 & & toward tunnel & 7 & 4.7 \\
\hline R148 & & toward solvent & 7 & N.A. \\
\hline D149 & & toward solvent & 9 & N.A. \\
\hline L150 & & toward solvent & 9 & N.A. \\
\hline F151 & & toward tunnel & 6 & 8.4 \\
\hline F154 & & along tunnel & 6 & N.A. \\
\hline F169 & \multirow{6}{*}{ along tunnel } & toward tunnel & 2 & N.A. \\
\hline Q172 & & toward solvent & 9 & N.A. \\
\hline V173 & & toward tunnel & 5 & N.A. \\
\hline L174 & & to protein core & 4 & N.A. \\
\hline G176 & & toward solvent & 9 & N.A. \\
\hline L177 & & toward tunnel & 6 & N.A. \\
\hline G246 & \multirow{3}{*}{ across tunnel } & to protein core & 1 & N.A. \\
\hline A247 & & toward tunnel & 7 & 5.2 \\
\hline L248 & & along tunnel & 4 & N.A. \\
\hline A271 & \multirow{2}{*}{ along tunnel } & toward tunnel & 8 & N.A. \\
\hline $\mathrm{H} 272$ & & along tunnel & 1 & N.A. \\
\hline
\end{tabular}

$\mathrm{a}$ - location in the respect to the position $177 ; \mathrm{b}$ - calculated by Hotspot Wizard 1.7, score 1 represents highly conserved residues, whereas score 9 represents highly variable residues; c - distance measured from SG ( $\gamma$ sulfur) atom of introduced C177 to other introduced cysteines; mutants were constructed in PyMOL 1.7. N.A. - not applicable. The positions mutated in the LinB-Closed ${ }^{\mathrm{CC}}$ are shown in bold (see Figure S2). 
Table S3. Average geometries of nine potential pathways identified in 12 crystal structures of LinB-Wt.

CAVER tunnel ID Bottleneck radius $(\AA)$

Tunnel length $(\AA ̊)$

\begin{tabular}{cll}
$\mathbf{4}$ & $\mathbf{0 . 7 \pm 0 . 1}$ & $\mathbf{1 6 \pm 2}$ \\
5 & $0.8 \pm 0.1$ & $23 \pm 1$ \\
6 & $0.7 \pm 0.1$ & $23 \pm 2$ \\
7 & $0.7 \pm 0.1$ & $26 \pm 2$ \\
8 & $0.8 \pm 0.1$ & $26 \pm 2$ \\
9 & $0.8 \pm 0.1$ & $30 \pm 2$ \\
10 & $0.7 \pm 0.1$ & $32 \pm 1$ \\
13 & $0.7 \pm 0.1$ & $25 \pm 4$ \\
21 & $0.7 \pm 0.1$ & $45 \pm 4$ \\
\hline
\end{tabular}

The potential pathway selected for opening of the $\mathrm{p} 3$ tunnel is in bold. 
Table S4. Bottleneck residues of potential p3 pathway in LinB-Wt.

\begin{tabular}{ccc}
\hline Bottleneck & Residue & Mutability score $^{\text {a }}$ \\
\hline \multirow{3}{*}{ First } & F143 & $\mathbf{7}$ \\
& F151 & 5 \\
& P208 & 1 \\
I211 & $\mathbf{3}$ \\
\hline \multirow{3}{*}{ Second } & W140 & $\mathbf{7}$ \\
& R155 & 3 \\
& I213 & 5 \\
\hline
\end{tabular}

a - calculated by Hotspot Wizard 1.7, score 1 represents highly conserved residue, whereas score 9 represents highly variable residue; three residues selected for mutagenesis are highlighted in bold 
Table S5. Specific activities of LinB variants with engineered p3 pathway measured with a reduced set of six halogenated substrates.

\begin{tabular}{|c|c|c|c|c|c|c|c|}
\hline \multirow{2}{*}{ Enzyme } & \multirow{2}{*}{ Mutations } & \multicolumn{6}{|c|}{ Specific activities $\left(\mathrm{nmol} \cdot \mathrm{s}^{-1} \cdot \mathrm{mg}^{-1}\right)$} \\
\hline & & 1-bromobutane & 1-bromohexane & 1-iodobutane & 1,2-dibromoethane & 1,3-dibromopropane & bromocyclohexane \\
\hline LinB-Wt & N.A. & 48.9 & 29.3 & 56.5 & 133.4 & 925 & 40.5 \\
\hline LinB-Closed $^{c C}$ & $\mathrm{D} 147 \mathrm{C}+\mathrm{L} 177 \mathrm{C}$ & 10.3 & 9.5 & 9.0 & 8.2 & 1.4 & 28.9 \\
\hline LinB-M1 & D147C+L177C+F143L & 16.5 & 20.7 & 9.3 & 30.4 & 2.7 & 14.8 \\
\hline LinB-M2 & D147C+L177C+F143V & 18.9 & 22.6 & 11.6 & 26.9 & 1.4 & 25.1 \\
\hline LinB-M3 & $\mathrm{D} 147 \mathrm{C}+\mathrm{L} 177 \mathrm{C}+1211 \mathrm{~V}$ & 16.4 & 20.0 & 10.4 & 21.4 & 3.2 & 31.7 \\
\hline LinB-M4 & $D 147 C+L 177 C+W 140 L+1211 L$ & 9.4 & 12.2 & 6.8 & 37.8 & 5.8 & 5.6 \\
\hline LinB-M5 & $\mathrm{D} 147 \mathrm{C}+\mathrm{L} 177 \mathrm{C}+\mathrm{F} 143 \mathrm{Y}+1211 \mathrm{~L}$ & 15.8 & 10.5 & 12.7 & 69.1 & 11.7 & 9.5 \\
\hline LinB-M6 & $D 147 C+L 177 C+W 140 L+F 143 V+1211 L$ & 21.5 & 25.8 & 9.7 & 38.6 & 5.0 & 5.3 \\
\hline LinB-M7 & $D 147 C+L 177 C+W 140 L+F 143 Y+\mid 211 L$ & 23.8 & 9.6 & 19.2 & 77.9 & 26.2 & 14.4 \\
\hline LinB-M8 & $D 147 C+L 177 C+W 140 A+F 143 L+1211 L$ & 4.5 & 4.5 & 0.0 & 13.4 & 0.0 & 0.0 \\
\hline LinB-M9 & $\mathrm{D} 147 \mathrm{C}+\mathrm{L} 177 \mathrm{C}+\mathrm{W} 140 \mathrm{~L}+\mathrm{F} 143 \mathrm{~L}$ & 11.8 & 11.7 & 7.9 & 24.0 & 4.2 & 14.1 \\
\hline LinB-M10 & $\mathrm{D} 147 \mathrm{C}+\mathrm{L} 177 \mathrm{C}+\mathrm{W} 140 \mathrm{~T}+\mathrm{F} 143 \mathrm{~L}+\mathrm{I} 211 \mathrm{~L}$ & 19.6 & 19.6 & 8.6 & 40.7 & 5.6 & 7.6 \\
\hline LinB-M11 & $D 147 C+L 177 C+F 143 V+1211 L$ & 13.6 & 18.0 & 6.9 & 31.3 & 2.4 & 6.2 \\
\hline LinB-M12 & $\mathrm{D} 147 \mathrm{C}+\mathrm{L} 177 \mathrm{C}+\mathrm{F} 143 \mathrm{~L}+\mathrm{I} 211 \mathrm{~L}$ & 13.7 & 16.7 & 6.6 & 42.8 & 2.3 & 8.4 \\
\hline
\end{tabular}

Mutants selected for MALDI-MS/MS analysis are highlighted in bold. N.A. - not applicable 
Table S6 Specific activities of LinB variants with engineered p3 pathway measured with a set of 30 halogenated substrates.

\begin{tabular}{|c|c|c|c|c|c|c|}
\hline \multirow{2}{*}{$\begin{array}{l}\text { Substrate } \\
\text { code }\end{array}$} & \multirow{2}{*}{ Substrate } & \multicolumn{5}{|c|}{ Specific activities (nmol. $\mathrm{s}^{-1} \cdot \mathrm{mg}^{-1}$ ) } \\
\hline & & LinB-Wt & LinB-Closed $^{\mathrm{CC}}$ & LinB-Open $^{\text {CC }}$ & LinB-Closed $^{\mathrm{W}}$ & LinB-Open $^{W}$ \\
\hline 4 & 1-chlorobutane & 0.0231 & 0.0157 & 0.0067 & 0.0391 & 0.0127 \\
\hline 6 & 1-chlorohexane & 0.0270 & 0.0169 & 0.0182 & 0.0260 & 0.0163 \\
\hline 18 & 1-bromobutane & 0.0489 & 0.0103 & 0.0183 & 0.0365 & 0.0819 \\
\hline 20 & 1-bromohexane & 0.0293 & 0.0095 & 0.0188 & 0.0271 & 0.0733 \\
\hline 28 & 1-iodopropane & 0.0665 & 0.0091 & 0.0095 & 0.0273 & 0.1322 \\
\hline 29 & 1-iodobutane & 0.0565 & 0.0090 & 0.0061 & 0.0320 & 0.0422 \\
\hline 31 & 1-iodohexane & 0.0460 & 0.0084 & 0.0218 & 0.0279 & 0.0339 \\
\hline 37 & 1,2-dichloroethane & 0.0000 & 0.0063 & 0.0011 & 0.0082 & 0.0059 \\
\hline 38 & 1,3-dichloropropane & 0.0204 & 0.0097 & 0.0105 & 0.0299 & 0.0197 \\
\hline 40 & 1,5-dichloropentane & 0.0288 & 0.0084 & 0.0177 & 0.0297 & 0.0220 \\
\hline 47 & 1,2-dibromoethane & 0.1335 & 0.0082 & 0.0488 & 0.0184 & 0.2680 \\
\hline 48 & 1,3-dibromopropane & 0.0925 & 0.0014 & 0.0066 & 0.0205 & 0.1725 \\
\hline 52 & 1-bromo-3-chloropropane & 0.0860 & 0.0137 & 0.0201 & 0.0209 & 0.1326 \\
\hline 54 & 1,3-diiodopropane & 0.0479 & 0.0057 & 0.0073 & 0.0465 & 0.2590 \\
\hline 64 & 2-iodobutane & 0.0101 & 0.0088 & 0.0096 & 0.0102 & 0.0451 \\
\hline 67 & 1,2-dichloropropane & 0.0000 & 0.0000 & 0.0000 & 0.0059 & 0.0010 \\
\hline 72 & 1,2-dibromopropane & 0.0625 & 0.0333 & 0.0130 & 0.0177 & 0.0696 \\
\hline 76 & 2-bromo-1-chloropropane & 0.0599 & 0.0061 & 0.0350 & 0.0100 & 0.0548 \\
\hline 80 & 1,2,3-trichloropropane & 0.0000 & 0.0000 & 0.0000 & 0.0043 & 0.0018 \\
\hline 111 & bis(2-chloroethyl)ether & 0.0177 & 0.0080 & 0.0063 & 0.0017 & 0.0010 \\
\hline 115 & chlorocyclohexane & 0.0074 & 0.0000 & 0.0000 & 0.0046 & 0.0006 \\
\hline 117 & bromocyclohexane & 0.0249 & 0.0289 & 0.0083 & 0.0450 & 0.0390 \\
\hline 119 & (1-bromomethyl)cyclohexane & 0.0085 & 0.0025 & 0.0030 & 0.0504 & 0.0195 \\
\hline 137 & 1-bromo-2-chloroethane & 0.0940 & 0.0069 & 0.0374 & 0.0127 & 0.3525 \\
\hline 138 & chlorocyclopentane & 0.0059 & 0.0181 & 0.0072 & 0.0357 & 0.0260 \\
\hline 141 & 4-bromobutyronitrile & 0.0578 & 0.0051 & 0.0394 & 0.0489 & 0.1958 \\
\hline 154 & 1,2,3-tribromopropane & 0.0936 & 0.0210 & 0.0138 & 0.0473 & 0.0960 \\
\hline 155 & 1,2-dibromo-3-chloropropane & 0.0000 & 0.0171 & 0.0093 & 0.0263 & 0.0398 \\
\hline 209 & 3-chloro-2-methylpropene & 0.0351 & 0.0171 & 0.0095 & 0.0515 & 0.0781 \\
\hline 225 & 2,3-dichloropropene & 0.0155 & 0.0000 & 0.0089 & 0.0483 & 0.0397 \\
\hline
\end{tabular}


Table S7. Steady-state kinetics of LinB variants with substrate 1,2-dibromoethane.

\begin{tabular}{lcclcc}
\hline Enzyme & $\boldsymbol{k}_{\text {cat }}\left(\mathrm{s}^{-1}\right)$ & $\boldsymbol{K}_{\mathrm{m}}(\mathrm{mM})$ & $\boldsymbol{n}$ & $\boldsymbol{K}_{\mathrm{si}}(\mathrm{mM})$ & $\boldsymbol{k}_{\text {cat }} / \boldsymbol{K}_{\mathrm{m}}\left(\mathrm{mM}^{-1} \cdot \mathrm{s}^{-1}\right)$ \\
\hline LinB-Wt & $12 \pm 4$ & $1.7 \pm 0.2$ & N.A. & $13 \pm 0.6$ & $7 \pm 3$ \\
\hline LinB-Closed $^{\mathrm{cC}}$ & $2.1 \pm 0.2$ & $0.26 \pm 0.02$ & $1.65 \pm 0.05$ & $0.40 \pm 0.04$ & $8 \pm 1$ \\
LinB-Open $^{\mathrm{cc}}$ & $1.1 \pm 0.2$ & $0.71 \pm 0.03$ & $1.49 \pm 0.04$ & $23 \pm 5$ & $1.6 \pm 0.3$ \\
LinB-Closed $^{\mathrm{w}}$ & $3.1 \pm 0.1$ & $0.42 \pm 0.03$ & N.A. & $1.34 \pm 0.03$ & $7.3 \pm 0.6$ \\
LinB-Open $^{\mathrm{w}}$ & $57 \pm 3$ & $2.35 \pm 0.03$ & $1.11 \pm 0.01$ & $7.3 \pm 0.1$ & $24 \pm 1$
\end{tabular}

$\boldsymbol{k}_{\text {cat }}-$ turnover number, $\boldsymbol{K}_{\mathrm{m}}-$ Michaelis-Menten constant, $\boldsymbol{n}-$ Hill coefficient, $\boldsymbol{\kappa}_{\mathrm{si}}-$ substrate inhibition constant, $\boldsymbol{k}_{\mathrm{cat}} / \boldsymbol{K}_{\mathrm{m}}$ - catalytic efficiency; N.A. - not applicable 


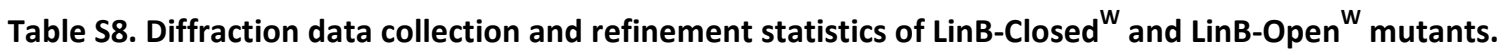

\begin{tabular}{|c|c|c|c|c|}
\hline \multirow{2}{*}{$\begin{array}{l}\text { Protein } \\
\text { PDB-ID }\end{array}$} & & \multirow{2}{*}{$\begin{array}{l}\text { LinB-Closed }^{W} \\
4 \mathrm{WDQ}\end{array}$} & \multicolumn{2}{|c|}{ LinB-Open $^{W}$} \\
\hline & & & 4WDR & 5LKA \\
\hline \multirow{14}{*}{$\begin{array}{l}\text { X-ray diffraction } \\
\text { data collection } \\
\text { statistics }\end{array}$} & Space group & $P 2_{1} 2_{1} 2_{1}$ & H32 & $P 2_{1} 2_{1} 2_{1}$ \\
\hline & Cell parameters $(\AA ̊ \cap, \stackrel{\circ}{)})$ & $a=46.7$ & $a=b=125.9$ & $a=46.87$ \\
\hline & & $b=68.5$ & $c=213.8$ & $b=68.36$ \\
\hline & & $c=81.1$ & $\alpha=\beta=90.0$ & $c=80.65$ \\
\hline & & $\alpha=\beta=\gamma=90.0$ & $\gamma=120.0$ & $\alpha=\beta=\gamma=90.0$ \\
\hline & Number of molecules in $\mathrm{AU}$ & 1 & 2 & 1 \\
\hline & Wavelength $(\AA ̊)$ & 0.8123 & 0.9724 & 0.9184 \\
\hline & Resolution ( $\AA$ ) & 1.58 & 2.50 & 1.30 \\
\hline & Number of unique reflections & 36,500 & 22,874 & 64,480 \\
\hline & Redundancy & 6.9 & 7.8 & 6.4 \\
\hline & Completeness (\%) & $98.3(85.2)$ & $99.7(98.7)$ & $99.6(98.7)$ \\
\hline & $R_{\text {merge }}{ }^{\mathrm{a}} / R_{\text {meas }}{ }^{\mathrm{b}}(\%)$ & $8.2(32.6)^{a}$ & $11.3(87.0)^{b}$ & $9.0(55.2)^{b}$ \\
\hline & Average $\mathrm{I} / \sigma(\mathrm{I})$ & $21.9(4.55)$ & $14.66(2.14)$ & $16.45(3.50)$ \\
\hline & Wilson $B\left(\AA^{2}\right)$ & 8.9 & 55.8 & 14.46 \\
\hline \multirow{11}{*}{$\begin{array}{l}\text { Refinement } \\
\text { statistics }\end{array}$} & Poclution rang $(\stackrel{1}{1}$ & $40.53-1.58$ & $48.58-2.50$ & $40.52-1.30$ \\
\hline & 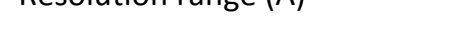 & $(1.63-1.57)$ & $(2.65-2.50)$ & $(1.32-1.30)$ \\
\hline & No. of reflections in working set & 34,335 & 21,730 & 62,366 \\
\hline & $R$ factor $(\%)^{c}$ & 16.2 & 16.7 & 13.9 \\
\hline & $R_{\text {free }}$ factor $(\%)^{c}$ & 20.6 & 19.6 & 17.5 \\
\hline & RMSD bond length (Å) & 0.012 & 0.014 & 0.006 \\
\hline & RMSD angle $\left({ }^{\circ}\right)$ & 1.399 & 1.602 & 0.921 \\
\hline & No. of atoms in $\mathrm{AU}$ & 3,126 & 4,673 & 2,988 \\
\hline & No. of protein atoms in $\mathrm{AU}$ & 2,407 & 4,609 & 2,531 \\
\hline & No. of water molecules in $\mathrm{AU}$ & 714 & 61 & 434 \\
\hline & Mean $B$ value all atoms $\left(\AA^{2}\right)$ & 12.4 & 59.0 & 13.45 \\
\hline Ramachandran & Residues in favored regions (\%) & $97.0[295 / 304]$ & $96.7[562 / 581]$ & $95.3[287 / 301]$ \\
\hline plot statistics & Residues in allowed regions (\%) & $100.0[304 / 304]$ & $100.0[581 / 581]$ & $100.0[301 / 301]$ \\
\hline
\end{tabular}

The data in parentheses refer to the highest-resolution shell.

${ }^{\mathrm{a}} R_{\text {merge }}=\Sigma_{\mathrm{hkl}} \Sigma_{\mathrm{i}} l_{i}(h k l)-\langle l(h k l)\rangle \mid / \Sigma_{\mathrm{hkl}} \Sigma_{\mathrm{i}} l_{i}(h k l)$, where $l_{i}(h k l)$ is the individual intensity of the ith observation of reflection $\mathrm{hkl}$ and $\langle l(h k l)\rangle$ is the average intensity of reflection $h k l$ with summation over all data.

${ }^{\mathrm{b}} R_{\text {meas }}$ is the redundancy-independent merging $R$ factor, also known as $R_{\text {r.i.m. }} \cdot R_{\text {meas }}=\Sigma_{h k l}[N /(N-1)]^{1 / 2} \Sigma_{\mathrm{i}}\left|l_{\mathrm{i}}(h k l)-\langle I(h k l)\rangle\right| / \Sigma_{h k l} \Sigma_{\mathrm{i}} l_{\mathrm{i}}(h k l)$, where $\langle l(h k l)\rangle$ is the mean of the $N(h k l)$ individual measurements $l_{i}(h k l)$ of the intensity of reflection $h k l$.

${ }^{\mathrm{c}} R$ factor $=|| \mathrm{F}_{\mathrm{o}}|-| \mathrm{F}_{\mathrm{c}}|| /\left|\mathrm{F}_{\mathrm{o}}\right|$, where $\mathrm{F}_{\mathrm{o}}$ and $\mathrm{F}_{\mathrm{c}}$ are the observed and calculated structure factors, respectively; the $R_{\text {free }}$ factor is calculated for the test set of reflections. 
Table S9. Geometry of the transport tunnels identified in the two crystal structures of LinB-Open ${ }^{w}$.

\begin{tabular}{|c|c|c|c|c|c|}
\hline PDB-I & & 4WDR - chain A & 4WDR - chain B & 5LKA & Average \\
\hline \multirow{4}{*}{$\begin{array}{c}\text { Bottleneck } \\
\text { radius }(\AA)\end{array}$} & p1b & 0.9 & 1.0 & 0.7 & $0.9 \pm 0.2$ \\
\hline & p1a & 0.8 & 1.0 & 0.7 & $0.9 \pm 0.2$ \\
\hline & p2 & 1.1 & 1.2 & 1.1 & $1.1 \pm 0.1$ \\
\hline & p3 & 1.1 & 1.0 & 1.0 & $1.0 \pm 0.1$ \\
\hline \multirow{4}{*}{ Length ( $\AA$ ) } & p1b & 14 & 13 & 21 & $16 \pm 4$ \\
\hline & p1a & 16 & 15 & 15 & $15 \pm 1$ \\
\hline & p2 & 20 & 16 & 20 & $19 \pm 2$ \\
\hline & p3 & 15 & 19 & 15 & $16 \pm 2$ \\
\hline
\end{tabular}


Table S10. Properties of transport tunnels analyzed over an ensemble of structures from molecular dynamics simulations of LinB variants.

\begin{tabular}{|c|c|c|c|c|c|}
\hline Enzyme & Tunnel & $\begin{array}{c}\text { Average bottleneck } \\
\text { (Å) }\end{array}$ & $\begin{array}{c}\text { Maximum bottleneck } \\
\text { (Å) }\end{array}$ & $\begin{array}{l}\text { Average length } \\
\text { (Å) }\end{array}$ & $\begin{array}{c}\text { Tunnel opening for } 1.4 \AA \\
\text { probe (\%) }\end{array}$ \\
\hline \multirow{4}{*}{ LinB-Wt } & $\mathrm{p} 1 \mathrm{~b}$ & $1.8 \pm 0.3$ & $2.5 \pm 0.1$ & $11 \pm 1$ & $89 \pm 9$ \\
\hline & $\mathrm{p} 1 \mathrm{a}$ & $1.6 \pm 0.1$ & $2.5 \pm 0.1$ & $13 \pm 1$ & $79 \pm 8$ \\
\hline & $\mathrm{p} 2$ & $1.2 \pm 0.1$ & $2.0 \pm 0.1$ & $21 \pm 1$ & $1 \pm 2$ \\
\hline & p3 & N.D. & N.D. & N.D. & N.D. \\
\hline \multirow{4}{*}{ LinB-Closed $^{\mathrm{W}}$} & $\mathrm{p} 1 \mathrm{~b}$ & $1.5 \pm 0.1$ & $2.4 \pm 0.1$ & $13 \pm 1$ & $70 \pm 10$ \\
\hline & $\mathrm{p} 1 \mathrm{a}$ & $1.4 \pm 0.1$ & $2.2 \pm 0.2$ & $14 \pm 1$ & $33 \pm 4$ \\
\hline & p2 & $1.0 \pm 0.1$ & $1.8 \pm 0.1$ & $22 \pm 2$ & $1 \pm 1$ \\
\hline & p3 & N.D. & N.D. & N.D. & N.D. \\
\hline \multirow{4}{*}{ LinB-Open ${ }^{w}$} & $\mathrm{p} 1 \mathrm{~b}$ & $1.4 \pm 0.1$ & $2.3 \pm 0.1$ & $12 \pm 1$ & $37 \pm 20$ \\
\hline & $\mathrm{p} 1 \mathrm{a}$ & $1.3 \pm 0.1$ & $2.2 \pm 0.1$ & $14 \pm 1$ & $18 \pm 12$ \\
\hline & $\mathrm{p} 2$ & $1.1 \pm 0.1$ & $2.1 \pm 0.2$ & $20 \pm 2$ & $2 \pm 1$ \\
\hline & p3 & $1.1 \pm 0.1$ & $2.0 \pm 0.2$ & $15 \pm 1$ & $2 \pm 1$ \\
\hline
\end{tabular}

N.D. - tunnel was not detected using a probe of $0.7 \AA$ radius in the molecular dynamics simulations with frequency $>0.1 \%$ 
Table S11. Utilization of tunnels for transport of water molecules during molecular dynamics simulations of LinB variants.

\begin{tabular}{|c|c|c|c|c|}
\hline \multirow{2}{*}{ Enzyme } & \multirow{2}{*}{ Tunnel } & \multicolumn{3}{|c|}{ Number of transported water molecules } \\
\hline & & Entry & Release & Total \\
\hline \multirow{4}{*}{ LinB-Wt } & $\mathrm{p} 1 \mathrm{~b}$ & $1,684 \pm 219$ & $1,672 \pm 202$ & $3,356 \pm 420$ \\
\hline & $\mathrm{p} 1 \mathrm{a}$ & $944 \pm 227$ & $950 \pm 226$ & $1,893 \pm 453$ \\
\hline & p2 & $70 \pm 50$ & $65 \pm 54$ & $135 \pm 102$ \\
\hline & p3 & $3 \pm 3$ & $3 \pm 4$ & $6 \pm 6$ \\
\hline \multirow{4}{*}{ LinB-Closed $^{\mathrm{W}}$} & $\mathrm{p} 1 \mathrm{~b}$ & $841 \pm 75$ & $838 \pm 66$ & $1,679 \pm 140$ \\
\hline & p1a & $152 \pm 26$ & $146 \pm 16$ & $298 \pm 39$ \\
\hline & $\mathrm{p} 2$ & $28 \pm 10$ & $29 \pm 15$ & $57 \pm 25$ \\
\hline & p3 & $2 \pm 3$ & N.D. & $2 \pm 3$ \\
\hline \multirow{4}{*}{ LinB-Open ${ }^{w}$} & $\mathrm{p} 1 \mathrm{~b}$ & $593 \pm 221$ & $573 \pm 201$ & $1,167 \pm 422$ \\
\hline & $\mathrm{p} 1 \mathrm{a}$ & $186 \pm 108$ & $198 \pm 122$ & $384 \pm 228$ \\
\hline & $\mathrm{p} 2$ & $27 \pm 12$ & $30 \pm 8$ & $56 \pm 17$ \\
\hline & p3 & $17 \pm 8$ & $16 \pm 6$ & $33 \pm 13$ \\
\hline
\end{tabular}

N.D. - no water transport was observed within replicated molecular dynamics simulations. 
Table S12. Product release pathways observed in accelerated molecular dynamics simulations of LinB variants.

\begin{tabular}{|c|c|c|c|c|c|c|}
\hline \multirow{2}{*}{ Enzyme } & \multirow{2}{*}{$\begin{array}{l}\text { Number of } \\
\text { simulations }\end{array}$} & \multirow{2}{*}{ Product } & \multicolumn{4}{|c|}{ Employed tunnel } \\
\hline & & & p1b & p1a & p2 & p3 \\
\hline \multirow{2}{*}{ LinB-Wt } & 100 & bromide ion & $38 \%(38)$ & $60 \%(60)$ & $2 \%(2)$ & $0 \%(0)$ \\
\hline & & 2-bromoethanol & $6 \%(6)$ & $79 \%(79)$ & $12 \%(12)$ & $3 \%(3)$ \\
\hline \multirow{2}{*}{ LinB-Closed $^{w}$} & 99 & bromide ion & $37 \%(37)$ & $62 \%(61)$ & $1 \%(1)$ & $0 \%(0)$ \\
\hline & & 2-bromoethanol & $9 \%(9)$ & $75 \%(74)$ & $11 \%(11)$ & $5 \%(5)$ \\
\hline \multirow{2}{*}{ LinB-Open $^{w}$} & 285 & bromide ion & $34 \%(97)$ & $60 \%(171)$ & $3 \%(9)$ & $3 \%(8)$ \\
\hline & & 2-bromoethanol & $12 \%(35)$ & $58 \%(165)$ & $17 \%(48)$ & $13 \%(37)$ \\
\hline
\end{tabular}

The frequency at which product release along each tunnel was observed in accelerated molecular dynamics simulations is given as a percentage and the corresponding number is shown in parenthesis. 